\title{
Influence of spatially variable ground heat flux on closed-loop geothermal systems: Line source model with nonhomogeneous Cauchy-type top boundary conditions
}

\author{
Jaime A. Rivera, ${ }^{\mathrm{a},{ }^{*}}$, Philipp Blum ${ }^{\mathrm{b}}$, Peter Bayer $^{\mathrm{a}}$ \\ ${ }^{a}$ ETH Zurich, Department of Earth Sciences, Sonneggstrasse 5, 8092 Zurich, Switzerland \\ ${ }^{\mathrm{b}}$ Karlsruhe Institute of Technology (KIT), Institute for Applied Geosciences (AGW), Kaiserstraße 12, \\ 76131 Karlsruhe, Germany \\ *Corresponding author. Tel.: +41-44-6332594, fax: +41-44-6331108. E-mail address: \\ jaime.rivera@erdw.ethz.ch
}

Keywords: ground heat exchange; ground surface temperature; shallow geothermal energy; land use change, urban environment. 


\begin{abstract}
Borehole heat exchangers (BHEs) utilize the shallow ground to extract geothermal energy. Mostly they are installed in urbanized areas, where the thermal regime is strongly influenced by pavements, buildings and other urban infrastructures. In order to account for the spatial and temporal variability in the above-ground urban temperatures, a new semi-analytical model with a Cauchy-type top boundary is introduced. With this model, it is possible to estimate the transient three-dimensional temperature field in the near-surface ground influenced by the interaction of BHEs, horizontal groundwater flow, land use type and associated surface air temperature (SAT). It is verified with a numerical model and sensitivity analyses are conducted to examine the relevance of the prevailing thermal regime. By adopting a dimensionless formulation, it is shown that the decoupling between temperature fields at the ground surface restraints heat fluxes and penetration depth of thermal signals above ground. A systematic comparison with traditional Dirichlet-type boundary conditions shows that a fixed temperature formulation generally overestimates the thermal effect of land surface signals on thermal plumes of BHEs. This is also addressed by investigating the ground energy balance during operation of the geothermal system.
\end{abstract}




\section{Nomenclature}

$A$

specific land use area $\left(\mathrm{m}^{2}\right)$

$a$

thermal diffusivity $\left(\mathrm{m}^{2} \mathrm{~s}^{-1}\right)$

$c$

volumetric heat capacity $\left(\mathrm{MJ} \mathrm{m}^{-3} \mathrm{~K}^{-1}\right)$

vertical fluxes at the ground surface $\left(\mathrm{W} \mathrm{m}^{-2}\right)$

F

dimensionless form of $f$

Fo

Fourier number

G

Green's function

$h$

coupling coefficient $\left(\mathrm{m}^{-1}\right)$

$H$

borehole length (m)

$H_{h}$

dimensionless product $H \cdot h$

I

linear heat transfer coefficient $\left(\mathrm{W} \mathrm{m}^{-2} \mathrm{~K}^{-1}\right)$

$k$

natural geothermal gradient $\left({ }^{\circ} \mathrm{C} \mathrm{m}^{-1}\right)$

$n_{\mathrm{e}}$

effective porous medium porosity

$p$

power (W)

$P$

dimensionless form of $p$

$P e$

Péclet number

D

Darcy velocity $\left(\mathrm{m} \mathrm{y}^{-1}\right)$

$Q_{T}$

$q$

heat flow rate per unit length $\left(\mathrm{W} \mathrm{m}^{-1}\right)$

$r_{d}$

horizontal radial distance from the borehole (m)

$R$

dimensionless form of $r_{d}$ 
$t$

$t_{\mathrm{o}}$

$T$

$T_{k}$

$T_{m}$

$T_{S}$

$T_{S A}$

$\overline{T_{S}}$

$v$

$x$

$x^{\prime}$

$x, y, z$

$x_{a}, x_{b}, y_{a}, y_{b}$

$x^{\prime}, y^{\prime}, z^{\prime}$

X

$X^{\prime}$

$X, Y, Z$

$X^{\prime}, Y^{\prime}, Z^{\prime}$

Greek symbols

$\beta$

B phase shift of $T_{S}$

time (s)

period of $T_{S}(\mathrm{~s})$

temperature in the porous medium $\left({ }^{\circ} \mathrm{C}\right)$

ground surface temperature corresponding to the geothermal gradient $k\left({ }^{\circ} \mathrm{C}\right)$

reference initial and surrounding temperature $\left({ }^{\circ} \mathrm{C}\right)$

above-ground temperature $\left({ }^{\circ} \mathrm{C}\right)$

amplitude of $T_{S}\left({ }^{\circ} \mathrm{C}\right)$

mean value of $T_{s}\left({ }^{\circ} \mathrm{C}\right)$

effective thermal velocity $\left(\mathrm{m} \mathrm{s}^{-1}\right)$

coordinates vector where temperature is evaluated $(\mathrm{m})$

coordinates vector where a heat source is released (m)

single space coordinates where temperature is evaluated $(\mathrm{m})$

boundary coordinates of the specific land use (m)

single space coordinates where heat sources are released (m)

dimensionless form of $\boldsymbol{x}(\mathrm{m})$

dimensionless form of $\boldsymbol{x}^{\prime}(\mathrm{m})$

dimensionless form of $x, y, z$

dimensionless form of $x^{\prime}, y^{\prime}, z^{\prime}$

solution to the homogeneous boundary-value problem

dimensionless form of $\beta$ 


\begin{tabular}{|c|c|}
\hline$\lambda$ & thermal conductivity $\left(\mathrm{W} \mathrm{m}^{-1} \mathrm{~K}^{-1}\right)$ \\
\hline$\kappa$ & substitution function \\
\hline $\mathrm{K}$ & dimensionless form of $\kappa$ \\
\hline$\tau$ & time at which a heat pulse is released (s) \\
\hline$\theta$ & dimensionless temperature \\
\hline$\varphi, \epsilon, E, \mu, M, \Delta, \rho, \sigma$ & intermediate or substitution variables \\
\hline$\omega$ & solution to the nonhomogeneous boundary-value \\
\hline$\Omega$ & dimensionless form of $\omega$ \\
\hline \multicolumn{2}{|l|}{ Subscripts } \\
\hline$\beta$ & referring to the HBVP $\beta$ \\
\hline$C$ & referring to Cauchy-type boundary conditions \\
\hline$D$ & referring to Dirichlet-type boundary conditions \\
\hline$\kappa, \mathrm{K}$ & expressed in terms of the functions $\kappa, \mathrm{K}$ \\
\hline$\omega$ & referring to the NHBVP $\omega$ \\
\hline$T$ & referring to the temperature $T$ \\
\hline \multicolumn{2}{|l|}{ Abbreviations } \\
\hline $\mathrm{BC}$ & boundary condition \\
\hline BHE & borehole heat exchanger \\
\hline GST & ground surface temperature \\
\hline HBVP & homogeneous boundary-value problem \\
\hline MFLS & moving finite line source \\
\hline NHBVP & nonhomogeneous boundary-value problem \\
\hline RS & reference scenario \\
\hline
\end{tabular}


surface air temperature

TDP temperature depth profile 


\section{Introduction}

Especially in urban areas, pristine natural land is rare and land surfaces are considerably modified. The strong heterogeneity in land use, together with micro-climatic conditions specific to individual cities and global climate trends [1], yields spatially and temporally variable thermal conditions above the ground surface and in the subsurface $[2,3]$. Implementation of numerical models for simulating heat transport across the ground surface is often a difficult task, mainly because of the multi-scale and spatiotemporal variability of parameters specifying the governing physical processes (e.g. [4-10]). Often, comprehensive numerical model development and the associated computational cost are not justified, given the quality and resolution of the data available to calibrate such models. Alternatives to this are simplified simulation techniques that focus on the most relevant processes, and which are on a par with the limited detail of case-specific information (e.g. [11-13]). These techniques can also be utilized to reveal, which key features should be explicitly resolved in more detailed models. In this context, analytical models have proven to be a keystone, not only because of their relatively simple implementation, but also because of their straightforward usability in parameter studies and sensitivity analyses.

Analytical models are widely accepted for simulating geothermal systems that use the shallow urban ground [14, 15], especially for sizing, optimizing and analysing vertical borehole heat exchangers (BHEs) [16-22]. Usually, these analytical models are based on the superposition of Green's functions [23, 24], which have been derived for several model configurations including line, spiral and cylindrical sources with (in-)finite lengths, with or without groundwater flow in (an-)isotropic media, or even considering phase-change [25]. Yet, available analytical models loosely consider the effect of complex top boundary conditions. Commonly, the temperature at the ground surface is assumed constant and is set equal to an initial temperature prevailing in the whole domain (e.g. [26, 27]). This, however, is unsatisfactory in view of the land use variability in urbanized areas and the associated impact on the heat transport across the ground surface.

Bandos et al. [28] relaxed the constant temperature assumption at the top boundary in an analytical framework for investigating the influence of seasonal ground surface temperature (GST) signals in thermal-response testing with BHEs. Rivera et al. [29] generalized this approach by implementing Green's functions to simulate specific features in urban environments. Their results show the potentially strong effect of long-term changes in GST on the thermal conditions around a BHE. Both models, by Bandos et al. [28] and Rivera et al. [29], assume a spatially and temporally variable GST implemented as $1^{\text {st }}$ kind or Dirichlet-type boundary condition (BC). However, a spatially resolved GST field is rarely available in 
practice. Best estimation is derived from punctual temperature-time series measured by sensors installed a few centimetres below the ground surface (e.g. [11, 30-32]). Therefore, as a surrogate, frequently the surface air temperature (SAT) is chosen (e.g. $[28,33,34])$, which is often available at a satisfactory spatial and temporal resolution. This assumes that the atmospheric and shallow ground thermal regime is perfectly coupled or in thermal equilibrium, which due to effects from wind, evapotranspiration, vegetative and snow insulation, among others, is not true $[35,36]$.

By formulating a $3^{\text {rd }}$ kind or Cauchy-type BC for the ground surface in a model, the temperature fields in atmosphere and ground can be coupled assuming a linear heat exchange rate [37]. This is still a simplified approach considering the complexity of the heat exchange mechanisms close to the ground surface $[38,39]$, but it is more versatile than fixed temperature assumptions, and it is generally acceptable within the range of accuracy expected from analytical models [40, 41]. Analytical models with Cauchy-type BCs were developed to estimate heat losses from isolated buildings [42, 43], or to understand the coupled dynamics between SAT and GST [44, 45]. These works though, do not study in-situ thermal changes by shallow geothermal applications, which were considered relevant in related numerical simulations (e.g. [38, 46, 47]).

Recently, Rivera et al. [48] incorporated the Cauchy-type boundary in a (semi-)analytical line source formulation for simulating BHEs. It was demonstrated that especially for short borehole heat exchangers (BHEs) the specification of the ground heat flux can be a crucial factor. While this formulation is one solution to the homogeneous boundary-value problem (HBVP), the present study introduces and investigates the nonhomogeneous problem (NHBVP). This way, nonuniform and transient land use changes characteristic for urban environments can be simulated in a flexible and efficient way. The new formulation is developed in the subsequent chapter and then verified with a numerical model. Furthermore, the objective is a detailed analysis of the relevance of appropriate SAT-GST (atmospheric or indoor-GST in basements of buildings) coupling for simulating the ground thermal regime around shallow geothermal installations. Thus, this study also addresses the role of the boundary formulation for groundwater influenced thermal plume development, for associated vertical heat fluxes, and for power supplied through the ground surface.

\section{Methodology}

In order to approximate the complex near surface processes in the ground, and their interaction with BHEs, this work proposes a general methodology based on the superposition of Green's 
functions. Specifically, defining a land surface-atmosphere/indoor coupling coefficient, $h$, the corresponding Green's function is given by [24]:

$$
\begin{aligned}
& G\left(\boldsymbol{x}^{\prime}, \boldsymbol{x}, t-\tau\right)= \\
& \frac{1}{8[a \pi(t-\tau)]^{\frac{3}{2}}}\left\{\exp \left[-\frac{\left(z-z^{\prime}\right)^{2}}{4 a(t-\tau)}\right]+\exp \left[-\frac{\left(z+z^{\prime}\right)^{2}}{4 a(t-\tau)}\right]\right\} \exp \left[-\frac{r_{d}^{2}}{4 a(t-\tau)}\right]- \\
& \frac{h}{4 a \pi(t-\tau)} \operatorname{erfc}\left[\frac{z+z^{\prime}}{2 \sqrt{a(t-\tau)}}+h \sqrt{a(t-\tau)}\right] \exp \left[h\left(z+z^{\prime}\right)+a h^{2}(t-\tau)-\frac{r_{d}^{2}}{4 a(t-\tau)}\right]
\end{aligned}
$$

where ${r_{d}}^{2}=\left(x-x^{\prime}\right)^{2}+\left(y-y^{\prime}\right)^{2}$. Eq. (1) yields the temperature at a given point $x=(x, y, z)$ and time $t$ in a semi-infinite homogeneous domain (with thermal diffusivity $a$ ), when an unitary heat pulse is released at $\boldsymbol{x}^{\prime}=\left(x^{\prime}, y^{\prime}, z^{\prime}\right)$ and time $\tau$. Here, $z$ represents the direction perpendicular to the semi-infinite boundary plane (depth). Eq. (1) is also the solution to the homogeneous boundary-value problem (HBVP) described in [48], where the medium above the ground (e.g. the atmosphere or indoor temperature) has a temperature of zero [49].

For a less restrictive temperature setting of the medium above ground, here the nonhomogeneous boundary-value problem (NHBVP) is considered. The solution to this problem, $\omega_{C}$, solves the conduction-dominated heat transport equation, assuming zero initial temperature and a boundary condition at the ground, as follows:

$$
\left.\lambda \frac{\partial \omega_{C}}{\partial z}\right|_{z=0}=-I\left(\omega_{C}-T_{S}\right) \quad \text { or }\left.\quad \frac{\partial \omega_{C}}{\partial z}\right|_{z=0}=-h\left(\omega_{C}-T_{S}\right) \text { with } h=\frac{I}{\lambda}
$$

where $T_{S}$ is the temperature of the medium above ground, which might be a function of space and/or time, $I\left[\mathrm{~W} \mathrm{~m}^{-2} \mathrm{~K}^{-1}\right]$ is the surface heat transfer coefficient, and $z$ points downwards and is normal to the ground surface. The coupling coefficient $h$ can vary between 0 and infinite, describing insulating and prescribed temperature conditions, respectively [24]. In other words, Newman and Dirichlet-type boundary conditions are specific extreme cases of this more general formulation.

The general solution to this NHBVP is obtained by superposition of continuous doublets distributed over the ground surface with an intensity proportional to $T_{s}\left(x^{\prime}, y^{\prime}, t\right)[24]$ : 


$$
\begin{aligned}
\omega_{C}\left(\boldsymbol{x}, \boldsymbol{x}^{\prime}, t\right)= & a \int_{0}^{t} \int_{\boldsymbol{x}^{\prime}} \int_{\boldsymbol{y}^{\prime}} T_{S}\left(x^{\prime}, y^{\prime}, \tau\right)\left(\frac{\partial G}{\partial z}\right) d y^{\prime} d x^{\prime} d \tau \\
& =a h \int_{0}^{t} \int_{x^{\prime}} \int_{y^{\prime}} T_{S}\left(x^{\prime}, y^{\prime}, \tau\right) \cdot G d y^{\prime} d x^{\prime} d \tau
\end{aligned}
$$

with $G$ as defined in Eq. (1). The solutions for both, homogeneous and nonhomogeneous boundary-value problems, can be superposed to solve more complex settings including arbitrary initial conditions and/or a non-uniform distribution of continuous heat sources within the domain. In this case, the temperature $T_{C}$ at any point and time reads:

$$
T_{C}=\omega_{C}+\beta_{C}
$$

where $\beta_{C}$ is the solution for the HBVP that could represent shallow geothermal systems such as BHEs, energy piles or coils. Specifically, if $\beta_{C}$ consists of a finite line source that approximates a BHE, this solution is developed in [48]:

$$
\beta_{C}=\beta_{M F L S}\left(x, x^{\prime}, t\right)+\Delta \beta_{C}\left(x, x^{\prime}, t\right)
$$

$\beta_{M F L S}\left(x, x^{\prime}, t\right)$

$$
\begin{aligned}
& =\frac{q}{8 \lambda \pi} \exp \left(\frac{x-x^{\prime}}{2 a} v\right) \int_{\frac{r_{d}^{2}}{4 a t}}^{\infty} \frac{1}{\varphi} \exp \left[-\varphi-\left(\frac{r_{d} v}{4 a}\right)^{2} \frac{1}{\varphi}\right]\left\{2 \operatorname{erf}\left(\frac{z}{r_{d}} \sqrt{\varphi}\right)\right. \\
& \left.-\operatorname{erf}\left(\frac{z-H}{r_{d}} \sqrt{\varphi}\right)-\operatorname{erf}\left(\frac{z+H}{r_{d}} \sqrt{\varphi}\right)\right\} d \varphi
\end{aligned}
$$

$\Delta \beta_{C}\left(\boldsymbol{x}, \boldsymbol{x}^{\prime}, t\right)$

$$
\begin{aligned}
& =\frac{q}{4 \lambda \pi} \exp \left(\frac{x-x^{\prime}}{2 a} v\right) \int_{\frac{r_{d}^{2}}{4 a t}}^{\infty} \frac{1}{\varphi} \exp (-\varphi \\
& \left.-\left(\frac{r_{d} v}{4 a}\right)^{2} \frac{1}{\varphi}\right)\left\{\operatorname { e x p } ( h z + ( \frac { h r _ { d } } { 2 } ) ^ { 2 } \frac { 1 } { \varphi } ) \left[\operatorname{erfc}\left(\frac{z \sqrt{\varphi}}{r_{d}}+\frac{h r_{d}}{2 \sqrt{\varphi}}\right)\right.\right. \\
& \left.\left.-\exp (h H) \operatorname{erfc}\left(\frac{z+H}{r_{d}} \sqrt{\varphi}+\frac{h r_{d}}{2 \sqrt{\varphi}}\right)\right]\right\} d \varphi
\end{aligned}
$$

Rivera et al. [48] also provides an alternative form for Eq. (7) to avoid potential numerical overflow while calculating the expression within the curly brackets. The problem might occur 
for a given combination of the parameters $h, z, r_{d}$ and $H$ leading to a large argument for the exponential function. This can be avoided via the following substitution:

$$
\begin{aligned}
\kappa\left(h, \mu, z, r_{d}\right) & \\
& =\frac{2}{r_{d}} \sqrt{\frac{\varphi}{\pi}} \int_{0}^{\infty} \exp \left[-h \varepsilon-\varphi\left(\frac{z+\mu+\varepsilon}{r_{d}}\right)^{2}\right] d \varepsilon \\
& =\exp \left[h z+h \mu+\left(\frac{h r_{d}}{2}\right)^{2} \frac{1}{\varphi}\right]\left[\operatorname{erfc}\left(\frac{z+\mu}{r_{d}} \sqrt{\varphi}+\frac{h r_{d}}{2 \sqrt{\varphi}}\right)\right]
\end{aligned}
$$

which leads to reformulate Eq. (7) as

$$
\begin{aligned}
\Delta \beta_{C}\left(\boldsymbol{x}, \boldsymbol{x}^{\prime}, t\right) & \\
& =\frac{q}{4 \lambda \pi} \exp \left(\frac{x-x^{\prime}}{2 a} v\right) \int_{\frac{r_{d}^{2}}{4 a t}}^{\infty} \frac{1}{\varphi} \exp \left[-\varphi-\left(\frac{r_{d} v}{4 a}\right)^{2} \frac{1}{\varphi}\right]\left[\kappa\left(h, 0, z, r_{d}\right)\right. \\
& \left.-\kappa\left(h, H, z, r_{d}\right)\right] d \varphi
\end{aligned}
$$

A triple integral such as in Eq. (3) compromises the computational efficiency of any analytical solution. This formulation can be simplified for the conceptual model shown in Figure 1. In this model, an area within the coordinates $\left[x_{a}, x_{b}\right]$ and $\left[y_{a}, y_{b}\right]$ frames a distinctive above-ground temperature $T_{s}$, which is a function of time. In the surrounding, zero temperature $\left(T_{m}=0\right)$ is assumed, which also prevails as initial condition in the conceptual model.

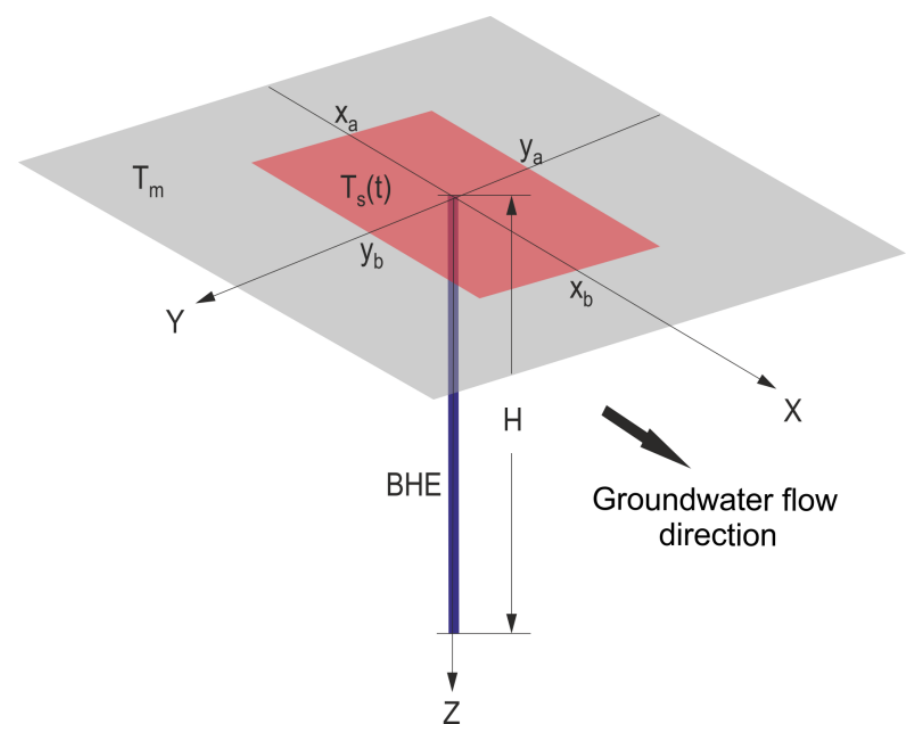

Figure 1: Conceptual model to represent spatial variability of land use in urban environments. The finite plane (red rectangle) within the coordinates $\left[x_{a}, x_{b}\right]$ and $\left[y_{a}, y_{b}\right]$ represents a distinctive above-ground temperature, $T_{S}$, in comparison to the background conditions, given by the mean reference temperature $T_{m}=0$. BHE denotes the position of the vertical borehole heat exchanger. 
For this configuration, Eq. (3) simplifies to the following expression including only one improper integral (Appendix A):

$$
\begin{aligned}
\omega_{C}(x, t)=\frac{H h}{16} & \int_{\frac{H^{2}}{4 a t}}^{\infty} T_{S}\left(t-\frac{H^{2}}{4 a \varphi}\right)\left\{\frac{2}{\sqrt{\pi \varphi^{3}}} \exp \left[-\left(\frac{z}{H}\right)^{2} \varphi\right]\right. \\
& \left.-\frac{H h}{\varphi^{2}} \operatorname{erfc}[\sigma(z, \varphi)] \exp [\rho(z, \varphi)]\right\} \Delta(x, \varphi) d \varphi, \quad z \neq 0
\end{aligned}
$$

where $\sigma(z, \varphi), \rho(z, \varphi)$ and $\Delta(\boldsymbol{x}, \varphi)$ are defined in Eqs. A5-A6 (Appendix A) and groundwater flow is considered via the moving source approach with effective thermal velocity $v=D \frac{c_{w}}{c}$. Thus, the Darcy flux $D$ is assumed uniform and steady [50].

The substitution shown in Eq. (8) facilitates rewriting Eq. (10) avoiding numerical overflow as:

$$
\begin{array}{r}
\omega_{C \kappa}(\boldsymbol{x}, t)=\frac{H h}{16} \int_{\frac{H^{2}}{4 a t}}^{\infty} T_{S}\left(t-\frac{H^{2}}{4 a \varphi}\right)\left\{\frac{2}{\sqrt{\pi \varphi^{3}}} \exp \left[-\left(\frac{z}{H}\right)^{2} \varphi\right]\right. \\
\left.-\frac{H h}{\varphi^{2}} \kappa(H h, 0, z / H, 1)\right\} \Delta(x, \varphi) d \varphi, \quad z \neq 0
\end{array}
$$

This set of equations yields the transient temperature distribution in the conceptual model in Figure 1. The latter, in turn, could be superposed in space (with or without the BHE) to approximate different spatial configurations of land use types.

For the case of an initial temperature that follows a natural geothermal gradient $k$ with ground surface temperature $T_{k}$, the following expression can be superposed in Eq. (5):

$$
\begin{array}{r}
\beta_{C o}=T_{k}+k z \cdot \operatorname{erf}\left(\frac{z}{\sqrt{4 a t}}\right)+2 k \sqrt{\frac{a t}{\pi}} \exp \left(-\frac{z^{2}}{4 a t}\right) \\
-h \int_{0}^{\infty} \kappa\left(h, z^{\prime}, z, \sqrt{4 a t \varphi}\right)\left(T_{k}+k z^{\prime}\right) d z^{\prime}
\end{array}
$$

Alternatively, in the simpler case of a constant initial temperature $T_{m} \neq 0$, the following analytical expressions is available in [24]:

$$
\beta_{C o}=T_{m}\left\{\operatorname{erf}\left(\frac{z}{\sqrt{4 a t}}\right)+\exp \left[h z+a t h^{2}\right] \operatorname{erfc}\left(\frac{z}{\sqrt{4 a t}}+h \sqrt{a t}\right)\right\}
$$


Finally, for the case of an above-ground temperature in the surrounding $T_{m}=T_{s}(t) \neq 0$, Eq. (10) or Eq. (11) should be superposed with $\left(x_{a}, x_{b}, y_{a}, y_{b}\right)$ going to the infinite. This yields the following analogous form shown in [24]:

$$
\begin{aligned}
\omega_{\mathrm{C} \_ \text {inf }}(z, t)= & a h \int_{0}^{t}\left\{\frac{\exp \left(-\frac{z^{2}}{4 a(t-\tau)}\right)}{\sqrt{\pi a(t-\tau)}}\right. \\
& \left.-h \exp \left[h z+a h^{2}(t-\tau)\right]\left[\operatorname{erfc}\left(\frac{z}{2 \sqrt{a(t-\tau)}}+h \sqrt{a(t-\tau)}\right)\right]\right\} T_{s}(\tau) d \tau
\end{aligned}
$$

Besides the estimation of temperature fields, another pertinent analysis is the characterization of heat fluxes and associated power supply through the ground surface in the conceptual model. For the homogeneous boundary-value problem, these fluxes are calculated directly by means of Eq. (2) with $T_{s}=0$ :

$$
f_{C \beta}=\left.\lambda \frac{\partial \beta_{C}}{\partial z}\right|_{z=0}=-h \lambda \beta_{C}
$$

For the nonhomogeneous boundary-value problem, we use Eq. (10) or Eq. (11) to calculate the corresponding vertical gradient at the ground surface:

$$
\begin{aligned}
f_{C \omega}=\lambda \frac{\partial \omega_{C}}{\partial z}= & \\
& =\frac{\lambda h}{16} \int_{\frac{H^{2}}{4 a t}}^{\infty} T_{S}\left(t-\frac{H^{2}}{4 a \varphi}\right)\left\{\frac{-4 z}{H \sqrt{\pi \varphi}} \exp \left[-\left(\frac{z}{H}\right)^{2} \varphi\right]\right. \\
& -\left(\frac{H h}{\varphi}\right)^{2} \exp [\rho(z, \varphi)] \operatorname{erfc}[\sigma(z, \varphi)] \\
& \left.+2 \sqrt{\frac{\varphi}{\pi}} \exp \left[\rho(z, \varphi)-\sigma(z, \varphi)^{2}\right]\right\} \Delta(x, \varphi) d \varphi, \quad z \neq 0
\end{aligned}
$$

At the ground surface, $f_{C \omega}$ can be approximated by evaluating Eq. (16) for values of $z$ close to zero, bearing in mind that this solution is not valid at $z=0$. Finally, the equation of total vertical heat flux reads as follows:

$$
f_{C T}(z=0, t)=f_{C \beta}(z=0, t)+f_{C \omega}(z \cong 0, t)
$$


The total power supplied through the ground surface can be calculated by integration of the fluxes over their influential area:

$$
\begin{aligned}
p_{C T}(z=0, t) & =\iint_{-\infty}^{\infty} f_{C \beta}(z=0, t) \cdot d x d y+\int_{-\infty}^{\infty} f_{C \omega}(z \cong 0, t) \cdot d x d y \\
& =p_{C \beta}(z=0, t)+p_{C \omega}(z \cong 0, t)
\end{aligned}
$$

The equation for $p_{C \beta}(z=0, t)$ is given in [48]:

$$
\begin{aligned}
p_{C \beta}(z=0, t) & \\
& =\frac{h q H^{2}}{4} \int_{\frac{H^{2}}{4 a t}}^{\infty} \frac{1}{\varphi^{2}} \exp \left[\left(\frac{H h}{2}\right)^{2} \frac{1}{\varphi}\right]\left[\operatorname{erfc}\left(\frac{H h}{2 \sqrt{\varphi}}\right)\right. \\
& \left.-\exp (H h) \operatorname{erfc}\left(\sqrt{\varphi}+\frac{H h}{2 \sqrt{\varphi}}\right)\right] d \varphi \\
& =\frac{h q H^{2}}{4} \int_{\frac{H^{2}}{4 a t}}^{\infty} \frac{1}{\varphi^{2}}[\kappa(h, 0,0, H)-\kappa(h, H, 0, H)] d \varphi
\end{aligned}
$$

For $p_{C \omega}(z \cong 0, t)$ we have (Appendix B):

$$
\begin{aligned}
p_{C \omega}(z \cong 0, t) & \\
& =\frac{\lambda h A}{4} \int_{\frac{H^{2}}{4 a t}}^{\infty} T_{s}\left(t-\frac{H^{2}}{4 a \varphi}\right)\left\{\frac{-4 z}{H \sqrt{\pi \varphi}} \exp \left[-\left(\frac{z}{H}\right)^{2} \varphi\right]\right. \\
& -\left(\frac{H h}{\varphi}\right)^{2} \exp [\rho(z, \varphi)] \operatorname{erfc}[\sigma(z, \varphi)] \\
& \left.+2 \sqrt{\frac{\varphi}{\pi}} \exp \left[\rho(z, \varphi)-\sigma(z, \varphi)^{2}\right]\right\} d \varphi
\end{aligned}
$$

with $A$ being the area of the rectangle in Figure 1. In order to highlight the effect of different boundary formulations, the presented set of equations for temperature (Eqs. (4) to (11)), vertical heat fluxes at the ground surface (Eqs. (15) to (17)), and associated power (Eqs. (18) to (20)), are subsequently compared with the analogous expressions assuming Dirichlet-type BCs as listed in Table 1. 
Table 1: Corresponding analytical formulations for Cauchy- and Dirichlet-type boundary conditions at the ground surface.

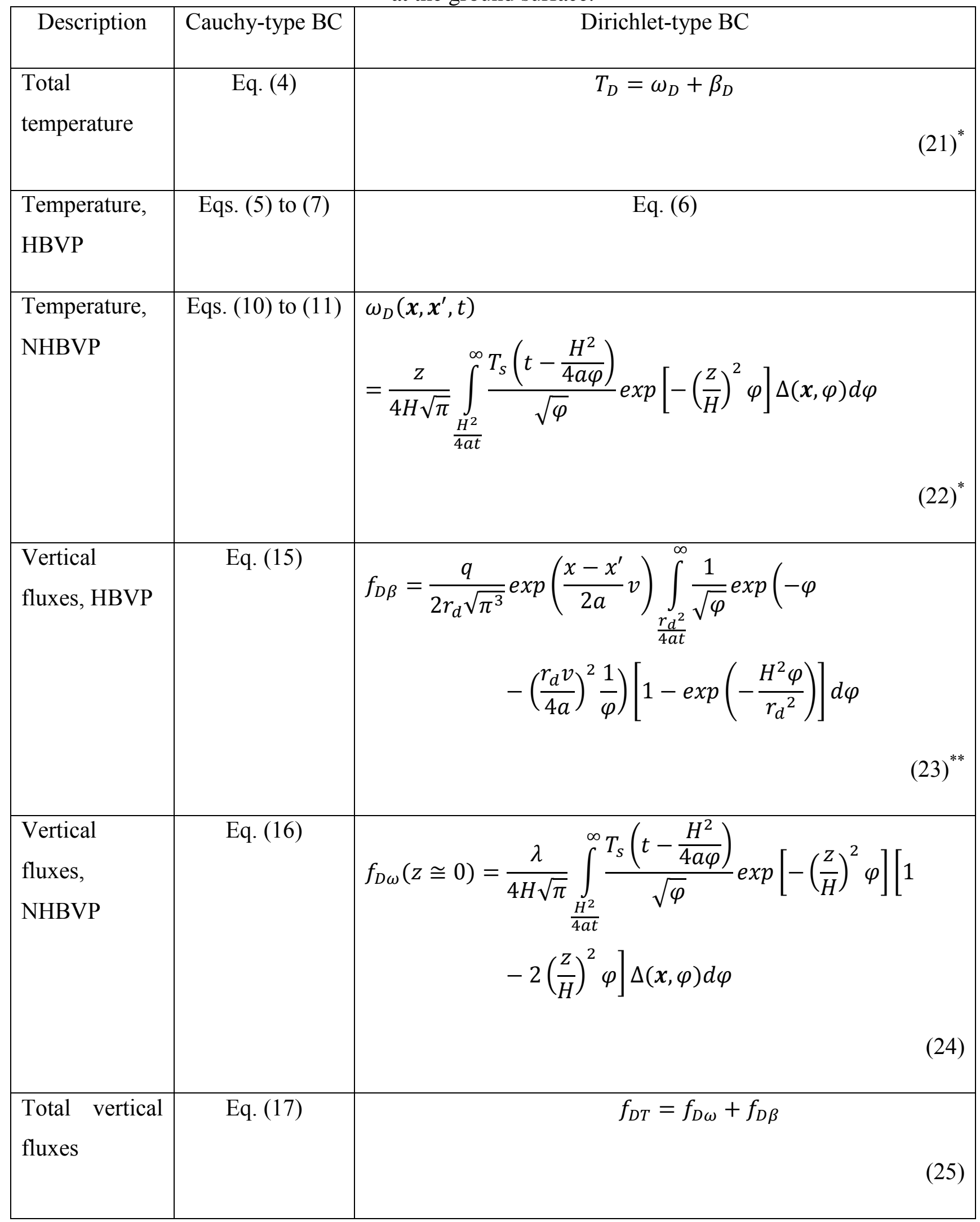




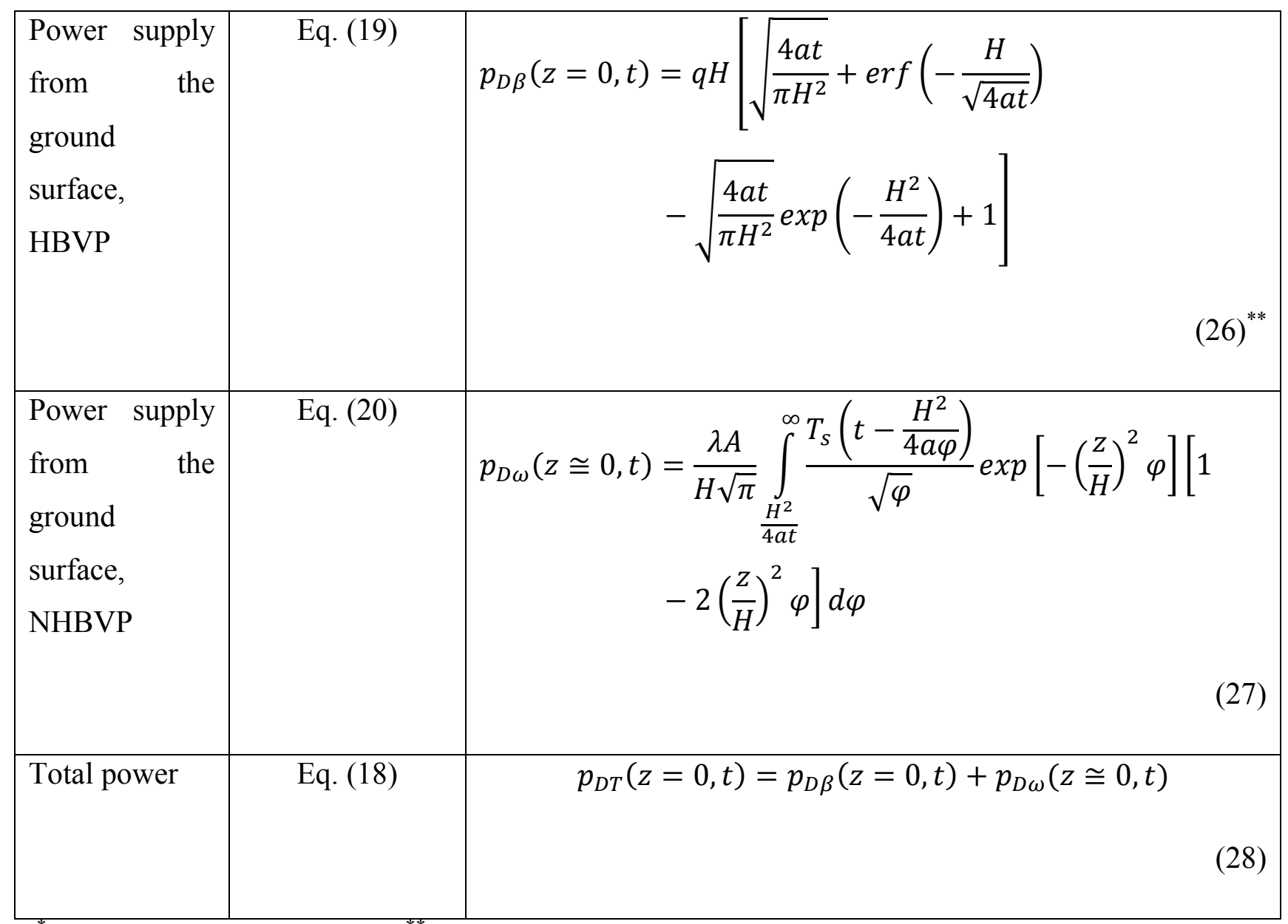

taken from [29], taken from ${ }^{* *}[51]$

In Table 1, Eq. (24) is obtained through the partial derivative of Eq. (22) with respect to $z$ and Eq. (27) by integrating Eq. (24) over the entire horizontal plane.

To generalize the comparison among different analytical formulations shown in Table 1, the following dimensionless numbers are considered:

$$
\begin{aligned}
& P e=\frac{v H}{a}, F o=\frac{a t}{H^{2}}, Q_{T}=\frac{q}{\lambda \bar{T}_{s}}, R=\frac{r_{d}}{H}, H_{h}=h H \\
& \left(X, X^{\prime}\right)=\left(<X, Y, Z>,<X^{\prime}, Y^{\prime}, Z^{\prime}>\right)=\frac{1}{H}\left(<x, y, z>,<x^{\prime}, y^{\prime}, z^{\prime}>\right) \\
& <X_{a}, X_{b}, Y_{a}, Y_{b}>=\frac{1}{H}<x_{a}, x_{b}, y_{a}, y_{b}>
\end{aligned}
$$

being $P e$ the Péclet number and $F o$ the Fourier number and $\overline{T_{S}}$ the mean value of $T_{S}$ which is assumed seasonally variable (Eq. A2 in Appendix A). All other coordinate-based parameters shown in the equations are normalized by the borehole length, $H$. The corresponding set of dimensionless equations and normalizing factors is listed in Appendix C. In the next chapter, the 
proposed analytical framework is first numerically verified. Subsequently, a sensitivity analysis based on the identified dimensionless groups is performed.

\section{Results and discussion}

\subsection{Numerical verification}

The model described by Eqs. (4) to (11) is verified with a numerical heat transport model (FEFLOW, Version 6.2). For this, the set-up shown in Figure 2 is considered.

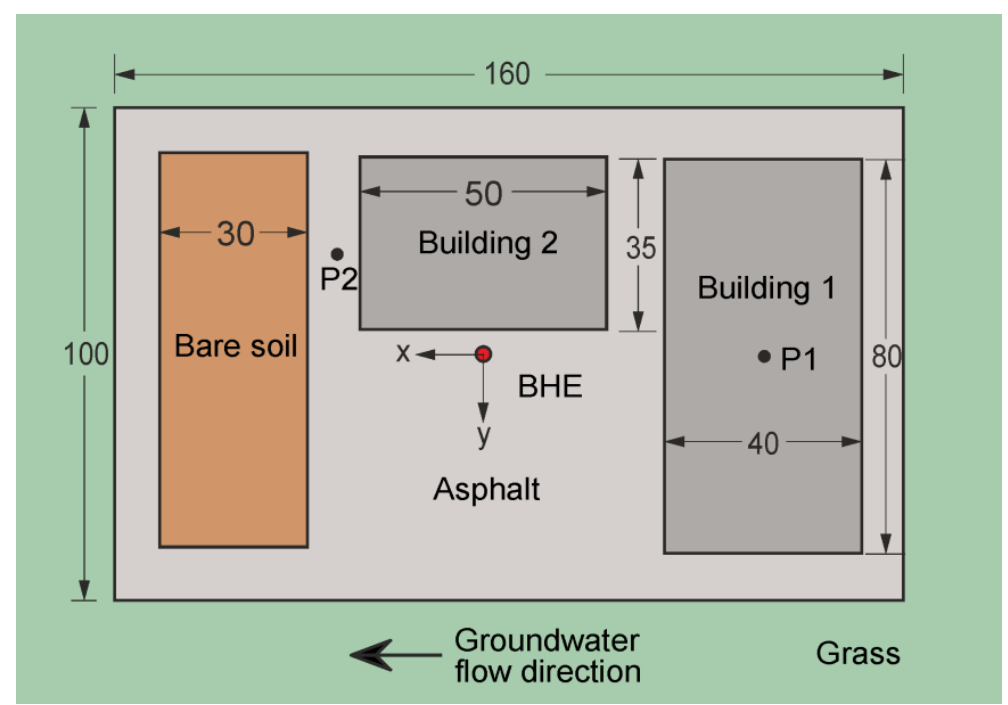

Figure 2: Plan view of the scenario with different land use types for verification with the numerical model. The borehole heat exchanger (BHE) is located at $(0,0)$. Spatial units are given in meters.

This model resembles the conditions in an urban environment with spatial changes in land use. For each land use type, the temperatures above-ground, $T_{s}$, are taken from $[11,12]$ following a seasonal behaviour with mean and amplitude as indicated in Table 2.

Table 2: Mean and amplitude temperature for above-ground temperatures $T_{s}$, given the set up shown in Figure 2 [11].

\begin{tabular}{|c|c|c|}
\hline Surface type & Mean temperature $\overline{T_{s}}\left({ }^{\circ} \mathrm{C}\right)$ & Amplitude $\left({ }^{\circ} \mathrm{C}\right)$ \\
\hline Building & $17.0^{*}$ & $0.0^{*}$ \\
\hline Asphalt & 13.2 & 18.6 \\
\hline Bare soil & 10.3 & 15.3 \\
\hline Grass & 10.1 & 13.1 \\
\hline
\end{tabular}

* Indoor building temperature taken from $[43,52]$ 
Other parameters in the physical model are taken from the numerical verification procedure described in [48] and summarized in Table 3.

Table 3: Parameters for the numerical verification model (Figure 2).

\begin{tabular}{|l|c|c|}
\hline Parameter & Units & Magnitude \\
\hline Darcy flux $(D)$ & $\mathrm{m} \mathrm{s}^{-1}$ & $1 \times 10^{-7}$ \\
\hline BHE length & $\mathrm{m}$ & 50 \\
\hline Heat extraction rate $q$ & $\mathrm{~W} \mathrm{~m}^{-1}$ & 30 \\
\hline Effective porosity $n_{e}$ & - & 0.25 \\
\hline $\begin{array}{l}\text { Bulk thermal conductivity of } \\
\text { porous medium } \lambda\end{array}$ & $\mathrm{W} \mathrm{m}^{-1} \mathrm{~K}^{-1}$ & 2.4 \\
\hline $\begin{array}{l}\text { Bulk volumetric heat capacity } \\
\text { of porous medium } c\end{array}$ & $\mathrm{MJ} \mathrm{K}^{-1} \mathrm{~m}^{-3}$ & 2.7 \\
\hline $\begin{array}{l}\text { Top boundary input/output } \\
\text { heat transfer coefficient } I\end{array}$ & $\mathrm{~W} \mathrm{~m}^{-2} \mathrm{~K}^{-1}$ & 1 \\
\hline
\end{tabular}

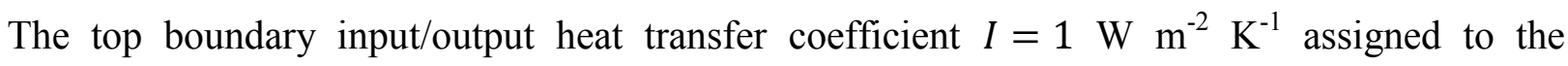
uppermost layer in the numerical model is equivalent to a coupling coefficient $h=I / \lambda=1 / 2.4=$ $0.4 \mathrm{~m}^{-1}$.

The size of the domain in the numerical model is chosen so that boundary effects are minimized during the simulation time of 30 years. The BHE is approximated by a linear discrete feature element (DFE) [53]. The domain discretization is refined close to the BHE and at the edges of different land use areas. The seasonal above-ground temperature signal for each land use type is approximated by linear segments in order to capture the major transient changes without stretching computational modelling time. In total, the model consists of more than 1.1 million nodes distributed in 141 layers with thicknesses ranging from $0.5 \mathrm{~m}$ close to the top boundary to $2 \mathrm{~m}$ at the bottom of the model. As initial condition, a uniform temperature equal to the mean temperature for grass $\left(10.10^{\circ} \mathrm{C}\right)$ was set.

For solving the same problem with the analytical framework, the conceptual model in Figure 1 is superposed as many times as the number of different land use areas. In each superposition, an effective above-ground temperature, $T_{s}$, is used for evaluating Eq. (10) or Eq. (11). As listed in

Table 4, this effective temperature is simply the difference between the one characteristic for the superposed land use type and the one assigned to the background. 
Table 4: Superposing conceptual models (Figure 1) according to the set-up shown in Figure 2.

\begin{tabular}{|c|c|c|}
\hline Land use type (area) & Effective $T_{s}$ in Eq. (10) & $\begin{array}{c}\text { BHE heat } \\
\text { extraction rate in } \\
\text { Eq. (5) }\end{array}$ \\
\hline Asphalt $(160 \times 100) \mathrm{m}^{2}$ & $T_{s}($ asphalt $)-T_{s}($ grass $)$ & $q=-30 \mathrm{~W} \mathrm{~m}^{-1}$ \\
\hline Building $1(80 \times 40) \mathrm{m}^{2}$ & $T_{s}($ building $)-T_{s}($ asphalt $)$ & 0 \\
\hline Building $2(50 \times 35) \mathrm{m}^{2}$ & $T_{s}($ building $)-T_{s}($ aspahlt $)$ & 0 \\
\hline Bare soil $(80 \times 30) \mathrm{m}^{2}$ & $T_{s}($ bare soil $)-T_{s}($ asphalt $)$ & 0 \\
\hline
\end{tabular}

The set of superimposed solutions listed in

Table 4 together with Eqs. (13) and (14) solves the problem depicted in Figure 2 at any point and time. The three-dimensional (3-D) temperature distribution after 20 years is shown in Figure 3. It is also worth mentioning that besides spatial heterogeneity in land use, the analytical solution can also handle their temporal variability such as the land use-change history typical for a selected city (e.g. $[54,55])$.



Figure 3: Temperature distribution after 20 years obtained with the analytical model for the scenario shown in Figure 2. The BHE is located at $(x, y)=(0,0)$ and groundwater flows along the positive $\mathrm{x}$ direction. 
Figure 4 compares two temperature depth profiles (TDPs) at the locations P1 (Figure 4a) and P2 (Figure 4b) indicated in Figure 2. The TDPs are calculated with both methodologies at four different times: 1, 2, 5 and 25 years. Only the upper $25 \mathrm{~m}$ are shown to highlight differences close to the ground surface.

In Figure 4a, the TDPs are not influenced by the operation of the BHE, since P1 is sufficiently far away at the upstream side. This supports the reliability of the NHBVP component $\left(\omega_{C}\right)$ in the total temperature and motivates its implementation for simulating the thermal conditions beneath large-scale urban areas as exemplified in Bayer et al. [56].

In contrast, the calculated TDPs in Figure 4b correspond to the point P2 located in an area influenced by multiple surface effects, groundwater flow and the cold plume generated by the BHE. This is properly reproduced. The highest differences between the analytical and numerical models are observed close to the BHE. This is plausible since the singularities associated to the analytical solution cannot be properly represented in a numerical model. Finally, Figure 4c depicts the calculated ground surface temperature (GST) at point P2 together with its surface air temperature (SAT for asphalt according to Figure 2). The clear difference between GST and SAT is induced by the coupling coefficient $h$. 

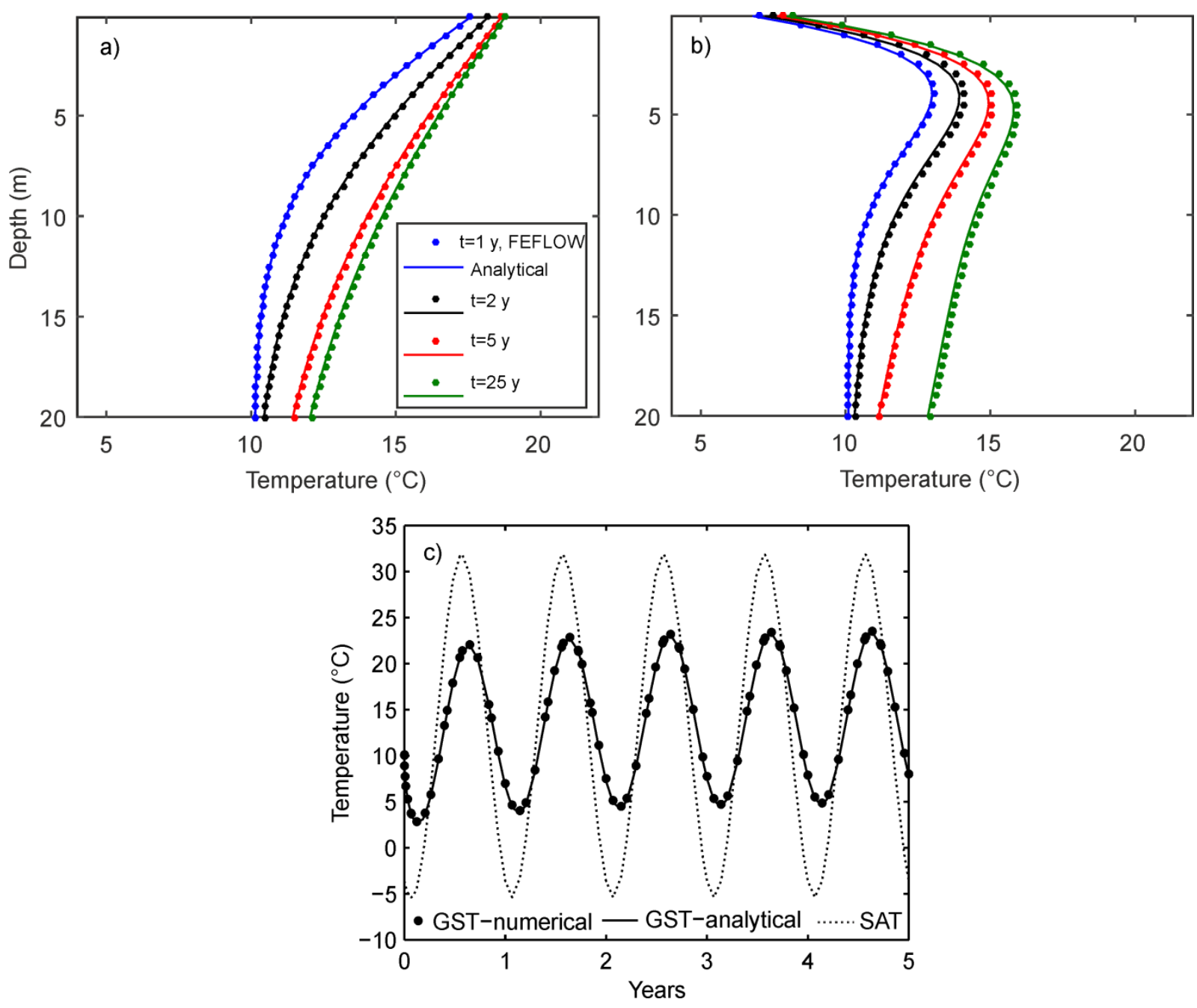

Figure 4: Analytically and numerically derived temperature-depth-profiles after 1, 2, 5 and 25 years, taken at the locations P1 (a) and P2 (b) as depicted in Figure 2 for the numerical verification exercise. In the plot (c) the calculated ground surface temperature (GST) at P2 is compared with surface air temperature, SAT.

\subsection{Effect of the boundary formulations on ground surface temperature (GST) and thermal plumes of BHEs}

The analysis described in [48] demonstrated that the implementation of Cauchy-type BCs, instead of simpler Dirichlet-type conditions, offers a better characterization of the in-situ thermal conditions near to the ground surface. The most notorious discrepancies are expected at the ground surface, where Dirichlet-type BCs do not reproduce the thermal interaction of the BHE with the above-ground medium. In the following, these discrepancies between both boundary formulations in case of a nonhomogeneous land use are quantified.

For keeping the analysis focused, we exclusively study the conceptual model depicted in Figure 1 and examine the effect of parameter variations. The properties of the porous medium are taken 
from the model set-up analysed in the previous section (Table 3). In this reference scenario, the BHE has a length of $H=100 \mathrm{~m}$ and a heat extraction rate of $q=-30 \mathrm{~W} \mathrm{~m}^{-1}$. The Darcy velocity is set to $D=4 \times 10^{-7} \mathrm{~m} / \mathrm{s}$ (representing a seepage velocity of around $50 \mathrm{~m} / \mathrm{y}$ ), and the reference coupling coefficient is $h=0.5 \mathrm{~m}^{-1}$. These magnitudes are the same as those discussed in [48] and define the reference dimensionless numbers as $P e=70$ and $H_{h}=50$.

For the temperature of the above-ground medium (red rectangle in Figure 1), it is assumed $T_{s}(t)=\overline{T_{s}}=1{ }^{\circ} \mathrm{C}$, so that unitary-based conclusions can be derived. For the compared analytical model based on Dirichlet-type BCs, $\overline{T_{s}}$ is the assumed prescribed temperature at the ground surface. In both cases, this temperature is applied to a square area on top with length equal to $H$.

The presented set of equations for either Dirichlet- or Cauchy-type BCs can provide the transient GST distribution given the settings of the conceptual model. However, to simplify the analysis, only steady-state conditions are considered. In fact, this state is reached relatively fast at the ground surface, and the GST behaves monotonically with time until steady-state is reached [48]. For this state, the dimensionless ground surface temperature (for Cauchy-type $\theta_{C}$ and for Dirichlet-type $\theta_{D}$ ) along the $x$ direction is shown in Figure 5. This is also the direction of groundwater flow. As indicated in the conceptual model (Figure 1), the BHE is located at $x=0$.
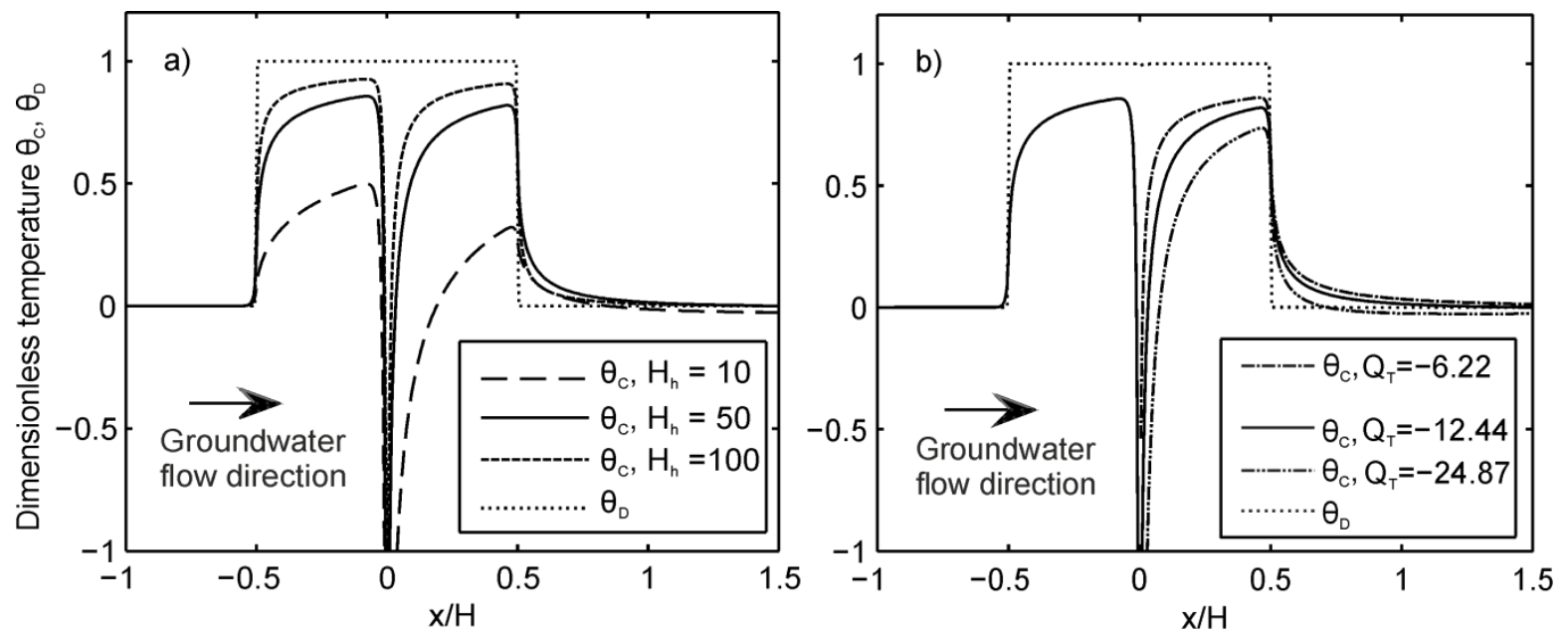

Figure 5: Dimensionless steady state GST for the conceptual model shown in Figure 1. Dirichlet$\left(\theta_{\mathrm{D}}\right)$ and Cauchy-type $\left(\theta_{\mathrm{C}}\right)$ BCs are compared changing (a) $H_{h}$ via the coupling coefficient $h$, and (b) $\mathrm{Q}_{\mathrm{T}}=\frac{\mathrm{q}}{\lambda \overline{\mathrm{T}_{\mathrm{s}}}}$ via the heat extraction rate $q$. The BHE is located at $x=0 \mathrm{~m}$.

In Figure 5a, the effect of the dimensionless group $H_{h}$ is analysed. Obviously, this number only influences $\theta_{C}$, since $\theta_{D}$ assumes a prescribed GST. Besides the reference $H_{h}=50$, the curves for $H_{h}=10$ and $H_{h}=100$ are calculated representing a higher insulating effect and a higher coupling 
behaviour at the ground surface, respectively. Figure 5a shows the trade-off between the two crucial parameters for computing the GST, $q$ and $\overline{T_{S}}$. Close to the origin, the contribution by $q$ is dominant, while the influence from $\overline{T_{s}}$ overprints the thermal sink in more distant areas. Groundwater flow yields an asymmetric temperature field with higher temperatures than the initial value downstream of the top plane $(X=x / H \geq 0.5)$. This feature, however, is not seen upstream of the same plane $(X \leq-0.5)$. As expected, for higher $H_{h}$ values, the curves of $\theta_{C}$ converge to the one for $\theta_{D}$, which corroborates the general relationship between Cauchy- and Dirichlet-type BCs at the ground surface.

In Figure 5b, the strengths of both $q$ and $\overline{T_{s}}$ are directly compared. For this, the dimensionless number $Q_{T}=\frac{q}{\lambda \bar{T}_{S}}$ is considered. In the reference scenario with $q=-30 \mathrm{~W} \mathrm{~m}^{-1}$ and $\overline{T_{s}}=1{ }^{\circ} \mathrm{C}$, its magnitude is $Q_{T}=-12.44$. For the two compared further scenarios we have $Q_{T}=-6.22$ and $Q_{T}=-24.87$, corresponding to extraction rates of -15 and $-60 \mathrm{~W} \mathrm{~m}^{-1}$, respectively. As revealed by Figure 5b, changing $Q_{T}$ via the value of $q$ only affects the downstream branch of the curves, since groundwater flow prevents upstream migration of the cold thermal plume. The observed lower ground surface temperatures for higher $Q_{T}$ is a self-explaining feature, but it is worth mentioning the relatively low sensitivity of the GST to changes in $q$ when compared with changes in $h$ (Figure 5a).

In Figure 6 the effect of the two boundary formulations are compared in the vertical profile. In order to highlight the model differences, steady-state conditions are assumed again and only the uppermost part of the domain is shown $(Z=z / H \leq 0.35)$. 

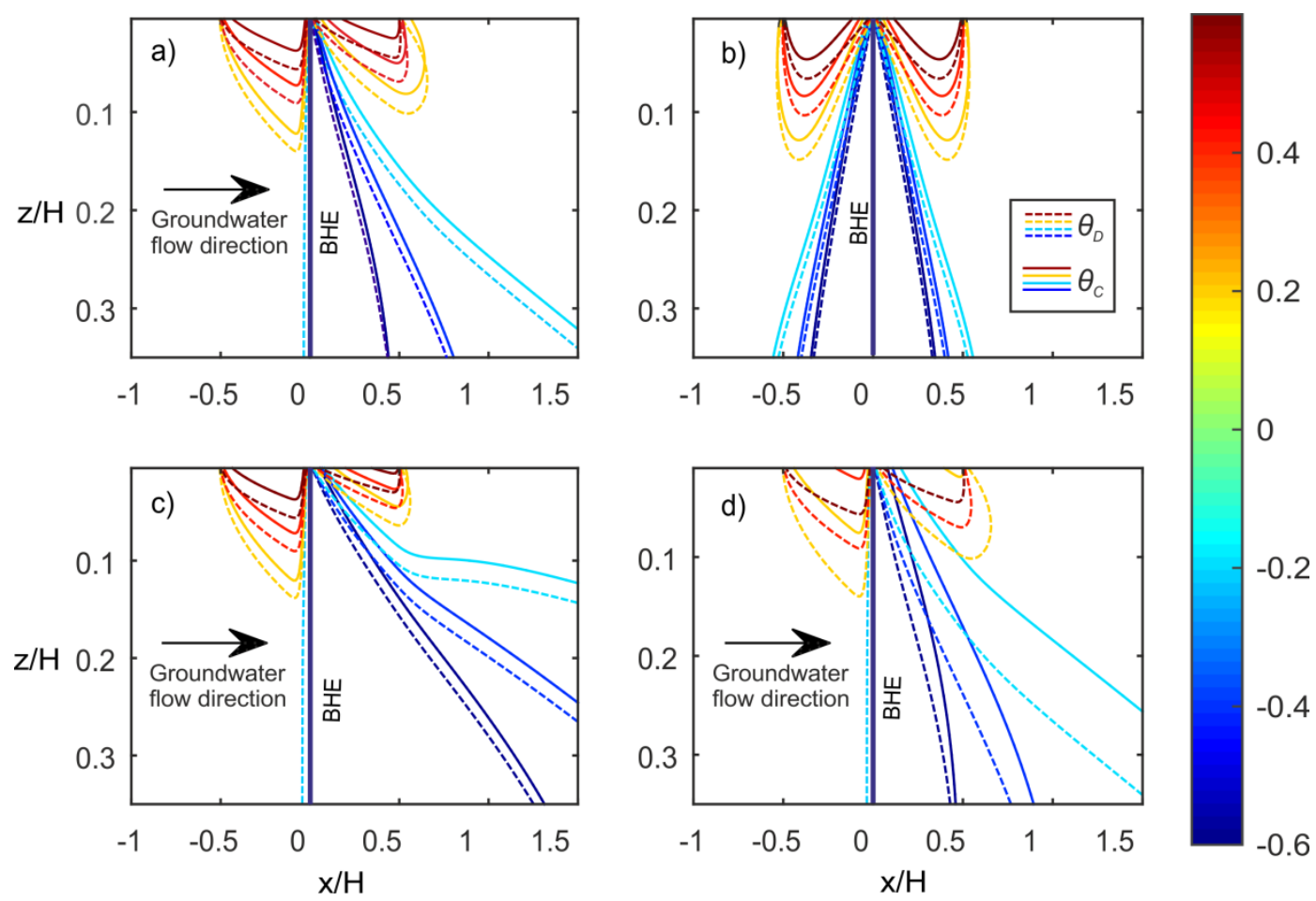

Figure 6: Dimensionless steady state temperature in the vertical plane $\mathrm{X}-\mathrm{Z}$ for the conceptual model shown in Figure 1. Dirichlet- $\left(\theta_{\mathrm{D}}\right)$ and Cauchy-type $\left(\theta_{\mathrm{C}}\right)$ BCs are compared in (a) the reference scenario (RS) with $H_{h}=50, P e=70$ and $\mathrm{Q}_{\mathrm{T}}=-12.44$, (b) RS with $P e=0$, (c) RS with $Q_{T}=-24.87$, and (d) RS with $H_{h}=10$. The BHE is located at $x=0$.

In Figure 6a, the result for the reference scenario (RS) is shown. In general, discrepancies between boundary formulations diminish with depth and close to the BHE where the strength of the line source overprints boundary effects. Rivera et al. [29] demonstrated that permanent changes in GST by urbanization effects can potentially influence thermal plumes in BHEs. Depending on the timing and strength of the GST signal, this influence can be seen even at the borehole toe. This analysis, however, was based on a model with prescribed GST. By changing the boundary formulation, Figure 6a indicates that, when SAT is taken as surrogate of GST, the penetration of the thermal signal on top may be overestimated. The lower penetration of this signal downstream of the BHE indicates again the interaction between groundwater flow, heat extraction $q$, and ground surface heat flux controlled by $\overline{T_{s}}$. It is important to bear in mind that the maximum downwards range of the signal on top (here up to $Z=0.15$ ) is computed here for $T_{S}(t)=\overline{T_{S}}=1{ }^{\circ} \mathrm{C}$. In urban environments, $\overline{T_{s}}$ can be (locally) several degrees above the mean background temperature $T_{m}$, for instance due to increased GST associated with paved streets and buildings $[11,12,57]$. Therefore, the penetration depth in cities can be much more significant. 
In Figure 6b, the thermal plumes are calculated for conditions without groundwater flow. Here the simulated discrepancies are enhanced, especially close to the top boundary. Also the penetration depth of the top signal is slightly deeper. Similar relationships were observed in [48]. At depths were the BHE becomes dominant, discrepancies are lower in the vicinity of the heat exchanger. In Figure 6c, the value of $Q_{T}$ is doubled by considering $q=-60 \mathrm{Wm}^{-1}$. Upstream, there are no differences when compared to the reference scenario. Downstream, discrepancies are amplified but not at the most shallow zone. In this case, the region with appreciable differences is most extended downstream due to the higher heat extraction rate. This stronger rate also mitigates the influence from the top signal.

In Figure 6d, the effect of $H_{h}$ is analysed. Compared with the reference $H_{h}=50$ in Figure $6 \mathrm{a}$, a higher insulating effect at the ground surface with $H_{h}=10$ is simulated. The higher degree of decoupling enhances the discrepancies between both models. In contrast to Figure $6 \mathrm{a}$, these discrepancies are now visible in the entire domain, however most notoriously close to the top boundary and far from the BHE.

\subsection{Effect of the boundary formulations on vertical heat fluxes through the ground surface}

For the same model set-up described in the previous section, Figure 7 depicts the normalized vertical heat fluxes through the ground surface and along the $x$-direction (which equals the groundwater flow direction). Negative fluxes indicate heat flow into the ground.

In all plots of Figure 7, the two dimensionless components (i.e. $F_{C \beta}$ and $F_{C \omega}$ ) of the total heat flux of the analytical solution based on Cauchy-type BC (Eq. (17)) are shown on the left axes. The absolute difference (for both flux components) to the solutions based on Dirichlet-type BC (Eq. (25)) is given on the right axes. 



Figure 7: Dimensionless steady-state vertical fluxes at the ground surface for the conceptual model shown in Figure 1. On the left axis, the homogeneous $\left(F_{C \beta}\right)$ and nonhomogeneous $\left(F_{C \omega}\right)$ solutions are compared. On the right axis, their absolute differences compared to calculation with Dirichlettype BC formulations $\left(F_{D \beta}, \mathrm{F}_{\mathrm{D} \omega}\right)$ are shown. (a) Reference scenario (RS) with $H_{h}=50, P e=70$ and $Q_{T}=-12.44$, (b) RS with $P e=0$, (c) RS with $Q_{T}=-24.87$, and (d) RS with $H_{h}=10$. The BHE is located at $x=0$; normalizing factor is $\frac{\mathrm{H}}{\lambda \overline{\mathrm{T}}_{\mathrm{S}}}$.

In Figure 7a, the dimensionless fluxes for the reference scenario are shown. Looking first at the fluxes for the NHBVP with Cauchy-type BC $\left(F_{C \omega}\right)$ and going from left to the right, an abrupt heat flux input at $\mathrm{X}=-0.5$ is seen, where $\mathrm{T}_{\mathrm{S}}$ changes from 0 to $1^{\circ} \mathrm{C}$. In the following, the fluxes decline, since the thermal gradient is less pronounced close to the origin (where the BHE is located). At $\mathrm{X}=x / H=0.5$, again an abrupt heat outflow appears due to the imposed condition of $\mathrm{T}_{\mathrm{s}}=0$ for $\mathrm{X} \geq 0.5$. Groundwater keeps this outflow of energy downstream until the system asymptotically reaches the undisturbed condition. In the case of the $\operatorname{HBVP}\left(F_{C \beta}\right)$, the maximum heat inflow is expected at the origin. Groundwater flow considerably reduces the heat fluxes upstream of the BHE', while it stimulates an extended heat input downstream. Similar behaviour was shown in [51] for BHEs simulated with Dirichlet-type BCs. Figure 7a also indicates that the magnitude (absolute value) of vertical fluxes induced by an unitary $\overline{T_{s}}$ is mostly higher than that induced by the BHE, except of in the range $0 \leq X \leq 0.5$. On the right 
(blue) axis of Figure 7a, the comparison between both boundary formulations indicates that discrepancies are only appreciable in areas where extreme gradients exist. In these areas, Dirichlet-based solutions, $F_{D \beta}$ and $F_{D \omega}$, have stronger magnitudes in absolute value since the boundary condition given by Eq. (2) smoothens the fluxes by constraining the thermal gradient. For completeness in this analysis, Figure 7 also includes the scenarios where the $P e$ is set to zero (Figure 7b) and the value of $Q_{T}$ is doubled (Figure 7c). In the first case, neglecting groundwater flow leads to a symmetric flux distribution with outflow of heat (upwards fluxes) outside of the square area on top $(|X| \geq 0.5)$. In the second case, doubling $Q_{T}$ (via $q$ ) only affects the homogeneous solutions $\left(F_{C \beta}\right.$ and $\left.F_{D \beta}\right)$, as can be concluded from the higher fluxes downstream when compared with the reference scenario. Keeping this reference, since the homogeneous solutions for both boundary formulations are linear with respect to $q$, there is no change in the difference $F_{D \beta}-F_{C \beta}$. A more interesting scenario is depicted in Figure $7 \mathrm{~d}$, where the dimensionless number $H_{h}$ is changed. In line with the previous analysis focused on the thermal plumes (Section 3.2), a higher insulation effect with $H_{h}=10$ is considered. By comparing with the reference scenario, in this case the heat fluxes $F_{C \beta}$ and $F_{C \omega}$ are clearly reduced and their distributions strongly smoothed. Consequently, the differences $F_{D \beta}-F_{C \beta}$ and $F_{D \omega}-F_{C \omega}$ associated with the right (blue) axis are enhanced.

\subsection{Effect of the boundary formulations on power supplied through the ground surface}

Hitherto, the analysis has been focused on the spatial variability of thermal plumes and heat fluxes while changing key parameters in the model. Yet, their temporal variability was not scrutinized in order to simplify the analysis, and therefore steady-state conditions were assumed. For the following total power analysis, however, the focus is the temporal dynamics, since this power is spatially independent for each land use. The total power is estimated by direct integration of the heat fluxes as described by Eq. (18) for the solution assuming Cauchy-type BC, and Eq. (28) if Dirichlet-type BCs are implemented. As noted above, these equations are not valid at $z=0$. However, the power supplied through the ground surface can be estimated taking a sufficiently small value for $z$, and thus simulating the conditions close to the ground surface.

One of the main characteristics of the equations for power estimation is that they are insensitive to groundwater flow. This independency of the overall power on the Pe number is due to the crucial assumptions of the moving source method as discussed in [51]. This insensitivity means that the groundwater flow only shapes the vertical fluxes (as shown in Figure 7a and Figure 7b), 
but the overall power remains the same as in conduction-dominating conditions. In other words, areas below the curves in Figure $7 \mathrm{a}$ and Figure $7 \mathrm{~b}$, are the same regardless of the value of $P e$.

To characterize this power, the same working scenario is discussed as in the previous sections. For the line source-based solutions (HBVP), [48] and [51] discuss in detail the power dynamics. Hence, the focus here is set on the $P_{C \omega}$ component. Figure 8 depicts these power magnitudes in dimensionless time $F o$, for the reference scenario with $Q_{T}=-12.44$. The red lines correspond to the NHBVP solution $P_{C \omega}(z \cong 0, t)$ (Eq. (20)) and the blue lines to the $\operatorname{HBVP} P_{C \beta}(z=0, t)$ (Eq. (19)). For each case, a set of curves is shown varying $H_{h}$ from 10 to infinite including the reference $H_{h}=50$. As previously, we set $T_{s}(t)=\overline{T_{s}}=1{ }^{\circ} \mathrm{C}, T_{m}=0{ }^{\circ} \mathrm{C}$ and $q=-30 \mathrm{~W} \mathrm{~m}^{-1}$. This means that the calculated power represents energy input to the ground. Here, it is also worth mentioning that, due to the spatial changes in $T_{S}$, some areas experience outflow of energy as indicated by the positive heat fluxes in Figure 7. Thus, the magnitudes shown in Figure 8 represent the net power effect, since $P_{C \omega}$ is based on flux integration over the entire ground surface.

The Fo numbers shown in Figure 8 span a time window of 60 years of BHE application for the reference scenario $(H=100 \mathrm{~m})$. In the beginning, the energy input from the finite plane on top (i.e. $\left.P_{C \omega}\right)$ is higher than the one stimulated by BHE operation (i.e. $P_{C \beta}$ ). At this stage, the condition of $T_{S}=1{ }^{\circ} \mathrm{C}$ creates a thermal gradient close to the ground that triggers this relatively high heat input. During the course of BHE operation, thermal equilibrium is reached leading to a systematic reduction of the difference between GST and $T_{s}$. This, in turn, restricts the power input and explains the decline of $P_{C \omega}$. At relatively late times, $P_{C \omega}$ also becomes insensitive to changes in $H_{h}$ and both boundary formulations asymptotically converge. For $P_{C \beta}$, this insensitivity is reached after a longer dimensionless time window. 


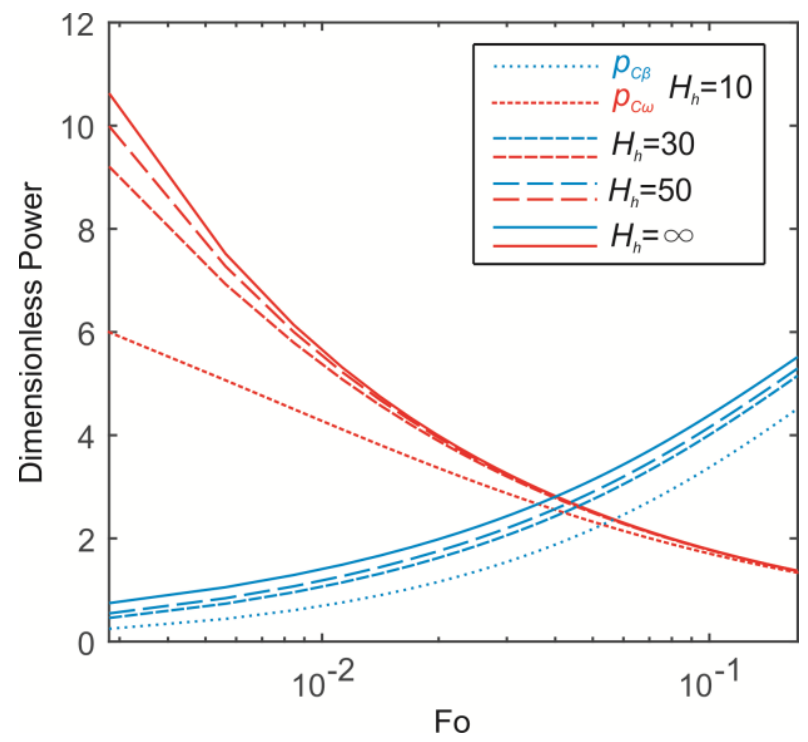

Figure 8: Dimensionless power for the conceptual model shown in Figure 1. Power contributions obtained by the nonhomogeneous $\left(\mathrm{P}_{\mathrm{C} \omega}\right)$ and homogenous $\left(\mathrm{P}_{\mathrm{C} \beta}\right)$ solutions are identified for different values of $H_{h}$. The normalizing factor is $\frac{\mathrm{H}}{\mathrm{A} \lambda \overline{\mathrm{T}_{\mathrm{s}}}}$.

The total power $P_{C T}$ is the superposition of $P_{C \omega}$ and $P_{C \beta}$. According to Figure 8 , a minimum of $P_{C T}$ is expected at around $F_{O}=0.04$. This point in time, however, also depends on the magnitudes of $Q_{T}$ and $H_{h}$, the difference in timing between changes in land use, and the starting of operating the BHE. In the long run, the total power is driven by its homogenous component $P_{C \beta}$, which is in accordance with $[48,51]$. Another conclusion from Figure 8 is, that taking for instance SAT as surrogate of GST might lead to an overestimation of the energy input to the ground. This is comparable with the deeper penetration of the signal on top when assuming Dirichlet-type BCs (i.e. $H_{h}=\infty$ ) as discussed in Figure 6.

The additivity of the analytical models for solving set-ups such as the one described in the numerical verification (Figure 2) enables to estimate transient power supply from different sources through the ground surface. Figure 9 shows the power (Figure 9a) and its associated energy (Figure 9b) for a time window of 60 years. For the BHE, also the power extracted from the reservoir is shown (BHE-Re). This power is calculated just by subtracting the power $P_{C \beta}$ from the total demand $q \cdot H$. The figure indicates that the energy individually provided by any of the buildings and from the asphalted ground can supply the energy taken from the reservoir during BHE operation. Moreover, the total energy input due to the overall land use change can feed several BHEs of same type. This is of course a theoretical evaluation of the geothermal potential, because the spatiotemporal distribution of the energy associated with land use changes is not necessarily connected with temporal changes of the thermal catchment volume around a 
BHE in the ground. Following this procedure, however, it would be possible to estimate the transient energy input from any densely urbanized area where timing, type and geometry of different land uses is known.
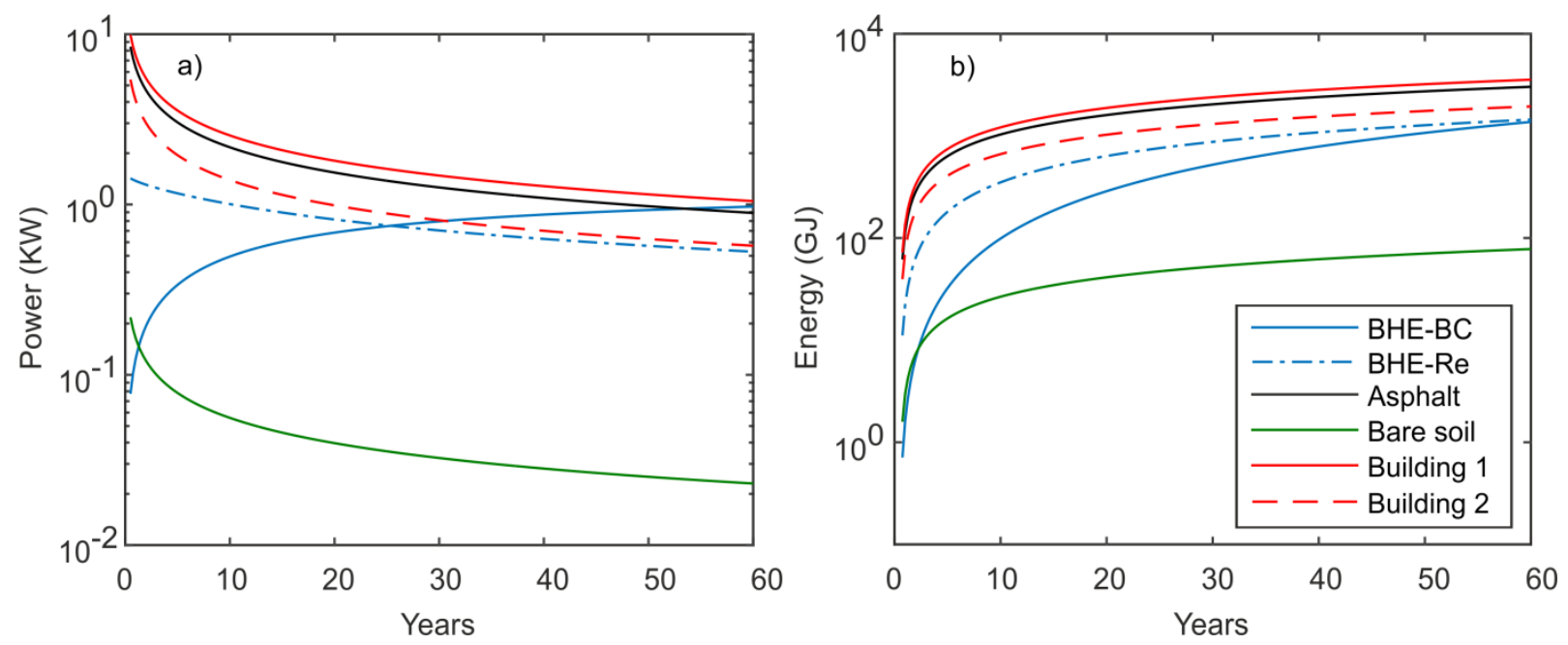

Figure 9: Power (a) and cumulative energy (b) supplied through the ground surface for the set-up shown in Figure 2. For the BHE, the power supplied by reservoir depletion (BHE-RE) and through the ground surface (BHE-BC) are identified.

\section{Conclusions}

In this work, a new (semi-)analytical solution for simulating the effect of above-ground temperatures on thermal plumes of BHEs is introduced. The applicability of the equation is scrutinized by means of a conceptual model that incorporates the spatial and temporal variability of surface (or indoor) temperatures as typical for urban environments. The boundary condition at the ground surface is formulated as Cauchy-type and by this, the heat fluxes are conditioned by the difference between the calculated ground surface temperature and a given above-ground temperature field. The presented model represents a solution to the nonhomogeneous boundaryvalue problem that can handle variability of land use types and associated ground heat fluxes. It is successfully verified with a numerical heat transport model.

The presented dimensionless analysis of the simulated ground thermal regime reveals the capability of the new solution to more realistically capture the near-surface thermal interaction between BHEs, surface air temperature, different land use types, and groundwater flow. It is demonstrated that taking an above-ground temperature field as an approximation of the ground surface temperature field may lead to an overestimation of these thermal effects in the ground. This is especially the case when horizontal groundwater flow is absent. When groundwater flow is included, the heat extraction rate $q$ of the BHE restricts the penetration of the ground surface thermal signal on the 
downstream side of the thermal plume. In general, even if ground heat flux is accounted for, the extraction rate represents the most important parameter in areas close to the BHE and at greater depth. If we distinguish regions in the ground based on the processes dominant for the thermal regime, these regions strongly depend on the decoupling degree and strength of the temperature fields interacting at the ground surface.

The analysis of ground vertical heat fluxes reveals that, besides the singularity associated with the position of the BHE, additional maxima or minima are expected at locations of land use change. In comparison to simulation with Dirichlet-type BCs, the formulation with Cauchy-type BCs introduces a smoothing effect on ground heat fluxes. This leads to a lower power input from the ground surface during the simulated time. The computed evolution of power input in the ground exhibits a divergent behaviour in the magnitudes induced by the operation of the BHE (homogenous boundary-value problem) and the ones generated by ground surface effects. While the former increases with time, the latter decreases and this yields a minimum in the transient trend of total power. The time, at which this minimum occurs, depends on specific properties of the analysed system.

As demonstrated by the synthetic study cases of this work, the superposition principle can be invoked to model complex settings in an efficient manner. This can be applied to field cases of any scale, such as local regimes around single BHEs, beneath residential districts or even cities. The presented procedure is also applicable to estimate the ground-surface power supply, for instance to estimate the theoretical and technical shallow geothermal energy potential in the ground [58].

\section{Acknowledgements}

This work was supported by the Swiss National Science Foundation (SNSF) under grant number 200021L 144288, and the German Research Foundation (DFG), under grant number BL 1015/4-1. 


\section{Appendix A:}

Substituting Eq. (1) in Eq. (3) for $z^{\prime}=0$ we have:

$$
\begin{aligned}
\omega_{C}\left(\boldsymbol{x}, \boldsymbol{x}^{\prime}, t\right)= & a h \int_{0}^{t} \int_{x^{\prime}} \int_{\boldsymbol{y}^{\prime}} T_{s}\left(x^{\prime}, y^{\prime}, \tau\right) \cdot G d y^{\prime} d x^{\prime} d \tau \\
& =a h \int_{0}^{t} \int_{x^{\prime}} \int_{y^{\prime}} T_{s}\left(x^{\prime}, y^{\prime}, \tau\right)\left\{\frac{2}{8[a \pi(t-\tau)]^{\frac{3}{2}}} \exp \left[-\frac{z^{2}}{4 a(t-\tau)}\right]\right. \\
& -\frac{h}{4 a \pi(t-\tau)} \operatorname{erfc}\left[\frac{z}{2 \sqrt{a(t-\tau)}}+h \sqrt{a(t-\tau)}\right] \exp [h z \\
& \left.\left.+a h^{2}(t-\tau)\right]\right\} \exp \left[-\frac{r_{d}^{2}}{4 a(t-\tau)}\right] d y^{\prime} d x^{\prime} d \tau
\end{aligned}
$$

For the conceptual model shown in Figure 1, we assume that the temperature $T_{s}\left(x^{\prime}, y^{\prime}, t\right)$ has the following seasonal form:

$$
T_{s}(t)=\overline{T_{s}}+T_{s A}\left\{\cos \left[2 \pi\left(\frac{t}{t_{o}}+s\right)\right]\right\}
$$

with mean temperature $\overline{T_{S}}$, amplitude $T_{S A}$, period $t_{o}$ and phase shift $s$. For this case, Eq. A1 can be written as:

$$
\begin{aligned}
\omega_{C}\left(\boldsymbol{x}, \boldsymbol{x}^{\prime}, t\right) & \\
& =a h \int_{0}^{t} T_{s}(\tau)\left\{\frac{2}{8[a \pi(t-\tau)]^{\frac{3}{2}}} \exp \left[-\frac{z^{2}}{4 a(t-\tau)}\right]\right. \\
& -\frac{h}{4 a \pi(t-\tau)} \operatorname{erfc}\left[\frac{z}{2 \sqrt{a(t-\tau)}}+h \sqrt{a(t-\tau)}\right] \exp [h z \\
& +a h^{2}(t \\
& -\tau)]\} \int_{x_{a}}^{x_{b}} \exp \left[-\frac{\left(x-x^{\prime}\right)^{2}}{4 a(t-\tau)}\right] d x^{\prime} \int_{x_{a}}^{x_{b}} \exp \left[-\frac{\left(y-y^{\prime}\right)^{2}}{4 a(t-\tau)}\right] d y^{\prime} d \tau
\end{aligned}
$$

The integrals over $\mathrm{x}^{\prime}$ and $\mathrm{y}^{\prime}$ can be directly evaluated yielding: 


$$
\begin{aligned}
\omega_{C}(\boldsymbol{x}, t)=a h & \int_{0}^{t} T_{S}(\tau)\left\{\frac{1}{4[a \pi(t-\tau)]^{\frac{3}{2}}} \exp \left[-\frac{z^{2}}{4 a(t-\tau)}\right]\right. \\
& -\frac{h}{4 a \pi(t-\tau)} \operatorname{erf}\left[\frac{z}{2 \sqrt{a(t-\tau)}}+h \sqrt{a(t-\tau)}\right] \exp [h z \\
& \left.\left.+a h^{2}(t-\tau)\right]\right\} \operatorname{ar}(t-\tau)\left[\operatorname{erf}\left(\frac{y-y_{b}}{4 a(t-\tau)}\right)\right. \\
& \left.-\operatorname{erf}\left(\frac{y-y_{a}}{4 a(t-\tau)}\right)\right]\left[\operatorname{erf}\left(\frac{x-x_{b}}{4 a(t-\tau)}\right)-\operatorname{erf}\left(\frac{x-x_{a}}{4 a(t-\tau)}\right)\right] d \tau
\end{aligned}
$$

An uniform groundwater flow velocity field $v$ can be incorporated by changing $x=x-$ $v(t-\tau)$. Furthermore, with the change of variable $\varphi=\frac{H^{2}}{4 a(t-\tau)}$ and the substitutions:

$$
\begin{gathered}
\Delta(\boldsymbol{x}, \varphi)=\left[\operatorname{erf}\left(\frac{y-y_{b}}{H} \sqrt{\varphi}\right)-\operatorname{erf}\left(\frac{y-y_{a}}{H} \sqrt{\varphi}\right)\right]\left[\operatorname{erf}\left(\frac{x-x_{b}}{H} \sqrt{\varphi}-\frac{v H}{4 a \sqrt{\varphi}}\right)\right. \\
\left.-\operatorname{erf}\left(\frac{x-x_{a}}{H} \sqrt{\varphi}-\frac{v H}{4 a \sqrt{\varphi}}\right)\right] \\
\sigma(z, \varphi)=\frac{z}{H} \sqrt{\varphi}+\frac{H h}{2 \sqrt{\varphi}} \\
\rho(z, \varphi)=z h+\frac{(H h)^{2}}{4 \varphi}
\end{gathered}
$$

the Eq. A4 becomes Eq. (10).

\section{Appendix B:}

For $P_{C \omega}(z \cong 0, t)$ from Eq. (18) we have:

$$
P_{C \omega}(z \cong 0, t)=\iint_{-\infty}^{\infty} f_{C \omega}(z \cong 0, t) \cdot d x d y
$$

Since $f_{C \omega}(z \cong 0, t)$ is given in Eq. (16) (including the substitutions A5 and A6) we have: 


$$
\begin{aligned}
P_{C \omega}(z \cong 0, t) & =\frac{\lambda h}{16} \iint_{-\infty}^{\infty} \int_{\frac{H^{2}}{4 a t}}^{\infty} T_{s}\left(t-\frac{H^{2}}{4 a \varphi}\right)\left\{\frac{-4 z}{H \sqrt{\pi \varphi}} \exp \left[-\left(\frac{z}{H}\right)^{2} \varphi\right]\right. \\
& -\left(\frac{H h}{\varphi}\right)^{2} \exp \left(z h+\frac{(H h)^{2}}{4 \varphi}\right)\left[\operatorname{erfc}\left(\frac{z}{H} \sqrt{\varphi}+\frac{H h}{2 \sqrt{\varphi}}\right)\right] \\
& +2 \sqrt{\frac{\varphi}{\pi}} \exp \left[z h+\frac{(H h)^{2}}{4 \varphi}\right. \\
& \left.\left.-\left(\frac{z}{H} \sqrt{\varphi}+\frac{H h}{2 \sqrt{\varphi}}\right)^{2}\right]\right\}\left[\operatorname{er}\left(\frac{y-y_{b}}{H} \sqrt{\varphi}\right)\right. \\
& \left.-\operatorname{erf}\left(\frac{y-y_{a}}{H} \sqrt{\varphi}\right)\right]\left[\operatorname{erf}\left(\frac{x-x_{b}}{H} \sqrt{\varphi}-\frac{v H}{4 a \sqrt{\varphi}}\right)\right. \\
& \left.-\operatorname{erf}\left(\frac{x-x_{a}}{H} \sqrt{\varphi}-\frac{v H}{4 a \sqrt{\varphi}}\right)\right] d \varphi d x d y
\end{aligned}
$$

Eq. B2 could be reorganized as follows:

$$
\begin{aligned}
P_{C \omega}(z \cong 0, t) & =\int_{\frac{H^{2}}{4 a t}}^{\infty} T_{s}\left(t-\frac{H^{2}}{4 a \varphi}\right)\left\{\frac{-4 z}{H \sqrt{\pi \varphi}} \exp \left[-\left(\frac{z}{H}\right)^{2} \varphi\right]\right. \\
& -\left(\frac{H h}{\varphi}\right)^{2} \exp \left(z h+\frac{(H h)^{2}}{4 \varphi}\right)\left[\operatorname{erfc}\left(\frac{z}{H} \sqrt{\varphi}+\frac{H h}{2 \sqrt{\varphi}}\right)\right] \\
& +2 \sqrt{\frac{\varphi}{\pi}} \exp \left[z h+\frac{(H h)^{2}}{4 \varphi}\right. \\
& \left.\left.-\left(\frac{z}{H} \sqrt{\varphi}+\frac{H h}{2 \sqrt{\varphi}}\right)^{2}\right]\right\} \int_{-\infty}^{\infty}\left[\operatorname{erf}\left(\frac{y-y_{b}}{H} \sqrt{\varphi}\right)\right. \\
& \left.-\operatorname{erf}\left(\frac{y-y_{a}}{H} \sqrt{\varphi}\right)\right] d y \int_{-\infty}^{\infty}\left[\operatorname{erf}\left(\frac{x-x_{b}}{H} \sqrt{\varphi}-\frac{v H}{4 a \sqrt{\varphi}}\right)\right. \\
& \left.-\operatorname{erf}\left(\frac{x-x_{a}}{H} \sqrt{\varphi}-\frac{v H}{4 a \sqrt{\varphi}}\right)\right] d x d \varphi
\end{aligned}
$$

The improper integrals over $x$ and $y$ in Eq. B3 are evaluated as follows:

$$
\begin{gathered}
\int_{-\infty}^{\infty}\left[\operatorname{erf}\left(\frac{y-y_{b}}{H} \sqrt{\varphi}\right)-\operatorname{erf}\left(\frac{y-y_{a}}{H} \sqrt{\varphi}\right)\right] d y=-2\left(y_{b}-y_{a}\right) \\
\int_{-\infty}^{\infty}\left[\operatorname{erf}\left(\frac{x-x_{b}}{H} \sqrt{\varphi}-\frac{v H}{4 a \sqrt{\varphi}}\right)-\operatorname{erf}\left(\frac{x-x_{a}}{H} \sqrt{\varphi}-\frac{v H}{4 a \sqrt{\varphi}}\right)\right] d x= \\
=-2\left(x_{b}-x_{a}\right)
\end{gathered}
$$


Substituting Eqs. B4 and B5 in Eq. B3, we obtain the Eq. (20).

\section{Appendix C:}

With the dimensionless numbers identified in Eq. (29), the following are the normalized forms of relevant equations:

\begin{tabular}{|c|c|c|}
\hline $\begin{array}{l}\text { Equation } \\
\text { in the } \\
\text { main text }\end{array}$ & Dimensionless form & $\begin{array}{l}\text { New } \\
\text { equation } \\
\text { id. }\end{array}$ \\
\hline (4) & $\theta_{C}=\Omega_{C}+\mathrm{B}_{C}$ & $\mathrm{C} 1$ \\
\hline (5) & $\mathrm{B}_{\mathrm{C}}=\left[\beta_{M F L S}\left(\boldsymbol{x}, \boldsymbol{x}^{\prime}, t\right)+\Delta \beta_{C}\left(\boldsymbol{x}, \boldsymbol{x}^{\prime}, t\right)\right] \frac{1}{\overline{T_{S}}}$ & $\mathrm{C} 2$ \\
\hline (6) & $\begin{aligned} \mathrm{B}_{M F L S}=\frac{\beta_{M F L S}}{\overline{T_{S}}} & \\
& =\frac{Q_{T}}{8 \pi} \exp \left(\frac{X P e}{2}\right) \int_{\frac{R^{2}}{4 F o}}^{\infty} \frac{1}{\varphi} \exp [-\varphi \\
& \left.-\left(\frac{R P e}{4}\right)^{2} \frac{1}{\varphi}\right]\left\{2 \operatorname{erf}\left(\frac{Z}{R} \sqrt{\varphi}\right)-\operatorname{erf}\left(\frac{Z-1}{R} \sqrt{\varphi}\right)\right. \\
& \left.-\operatorname{erf}\left(\frac{Z+1}{R} \sqrt{\varphi}\right)\right\} d \varphi\end{aligned}$ & $\mathrm{C} 3$ \\
\hline (7) & $\begin{aligned} \mathrm{B}_{C}=\frac{\Delta \beta_{C}}{\overline{T_{S}}}=\frac{Q_{T}}{4 \pi} & \exp \left(\frac{X P e}{2}\right) \int_{\frac{R^{2}}{4 F o}}^{\infty} \frac{1}{\varphi} \exp (-\varphi \\
& \left.-\left(\frac{R P e}{4}\right)^{2} \frac{1}{\varphi}\right)\left\{\exp \left(H_{h} Z\right.\right. \\
& \left.+\left(\frac{H_{h} R}{2}\right)^{2} \frac{1}{\varphi}\right)\left[\operatorname{erfc}\left(\frac{Z \sqrt{\varphi}}{R}+\frac{H_{h} R}{2 \sqrt{\varphi}}\right)\right. \\
& \left.\left.-\exp \left(H_{h}\right) \operatorname{erfc}\left(\frac{Z+1}{R} \sqrt{\varphi}+\frac{H_{h} R}{2 \sqrt{\varphi}}\right)\right]\right\} d \varphi\end{aligned}$ & $\mathrm{C} 4$ \\
\hline (10) & $\begin{aligned} \Omega_{C}=\frac{\omega_{C}}{\bar{T}_{s}}=\frac{H_{h}}{16} \int_{\frac{H^{2}}{4 a t}}^{\infty} \frac{T_{s}\left(t-\frac{H^{2}}{4 a \varphi}\right)}{\overline{T_{s}}}\left\{\frac{2}{\sqrt{\pi \varphi^{3}}} \exp \left[-Z^{2} \varphi\right]\right. \\
\left.\quad-\frac{H_{h}}{\varphi^{2}} \operatorname{erfc}\left(Z \sqrt{\varphi}+\frac{H_{h}}{2 \sqrt{\varphi}}\right) \exp \left(Z H_{h}+\frac{H_{h}{ }^{2}}{4 \varphi}\right)\right\} \Delta(\boldsymbol{X}, \varphi) d \varphi\end{aligned}$ & $\mathrm{C} 5$ \\
\hline
\end{tabular}




\begin{tabular}{|c|c|c|}
\hline$(8)$ & $\begin{aligned} \mathrm{K}\left(H_{h}, \mathrm{M}, Z, R\right) & =\kappa\left(h, \mu, z, r_{d}\right) \frac{H}{H} \\
= & \frac{2}{R} \sqrt{\frac{\varphi}{\pi}} \int_{0}^{\infty} \exp \left[-H_{h} \mathrm{E}-\varphi\left(\frac{Z+\mathrm{M}+\mathrm{E}}{R}\right)^{2}\right] d \mathrm{E}\end{aligned}$ & C6 \\
\hline (11) & $\begin{aligned} \Omega_{C \mathrm{~K}}=\frac{\omega_{C \mathrm{~K}}}{\bar{T}_{S}}= & \frac{H h}{16} \int_{\frac{H^{2}}{4 a t}}^{\infty} \frac{T_{s}\left(t-\frac{H^{2}}{4 a \varphi}\right)}{\overline{T_{s}}}\left\{\frac{2}{\sqrt{\pi \varphi^{3}}} \exp \left[-Z^{2} \varphi\right]\right. \\
& \left.-\frac{H_{h}}{\varphi^{2}} \mathrm{~K}\left(H_{h}, 0, Z, 1\right)\right\} \Delta(\boldsymbol{X}, \varphi) d \varphi\end{aligned}$ & C7 \\
\hline (15) & $F_{C \beta}=f_{C \beta} \frac{H}{\overline{T_{s}} \lambda}=-\frac{H_{h} \beta_{C}}{\overline{T_{s}}}=-H_{h} \mathrm{~B}_{C}$ & $\mathrm{C} 8$ \\
\hline (16) & $\begin{aligned} F_{C \omega}=f_{C \omega} \frac{H}{\overline{T_{s}} \lambda} & = \\
& =\frac{H_{h}}{16} \int_{\frac{1}{4 F o}}^{\infty} \frac{T_{s}\left(t-\frac{H^{2}}{4 a \varphi}\right)}{\overline{T_{s}}}\left\{\frac{-4 Z}{\sqrt{\pi \varphi}} \exp \left[-Z^{2} \varphi\right]\right. \\
& -\left(\frac{H_{h}}{\varphi}\right)^{2} \exp \left(Z H_{h}+\frac{{H_{h}}^{2}}{4 \varphi}\right)\left[\operatorname{erfc}\left(Z \sqrt{\varphi}+\frac{H_{h}}{2 \sqrt{\varphi}}\right)\right] \\
& \left.+2 \sqrt{\frac{\varphi}{\pi}} \exp \left[Z H_{h}+\frac{H_{h}{ }^{2}}{4 \varphi}-\left(Z \sqrt{\varphi}+\frac{H_{h}}{2 \sqrt{\varphi}}\right)^{2}\right]\right\} \Delta(\boldsymbol{X}, \varphi) d \varphi\end{aligned}$ & C9 \\
\hline (17) & $F_{C T}=F_{C \beta}+F_{C \omega}$ & $\mathrm{C} 10$ \\
\hline (18) & $P_{C T}=P_{C \beta}+P_{C \omega}$ & C11 \\
\hline (19) & $\begin{aligned} P_{C \beta}=p_{C \beta} \frac{H}{A \overline{T_{S}} \lambda} & \\
& =\frac{H_{h} Q_{T} H^{2}}{4 A} \int_{\frac{1}{4 F o}}^{\infty} \frac{1}{\varphi^{2}} \exp \left[\left(\frac{H_{h}}{2}\right)^{2} \frac{1}{\varphi}\right]\left[\operatorname{erfc}\left(\frac{H_{h}}{2 \sqrt{\varphi}}\right)\right. \\
& \left.-\exp \left(H_{h}\right) \operatorname{erfc}\left(\sqrt{\varphi}+\frac{H_{h}}{2 \sqrt{\varphi}}\right)\right] d \varphi\end{aligned}$ & $\mathrm{C} 12$ \\
\hline
\end{tabular}




\begin{tabular}{|c|c|c|}
\hline (20) & $\begin{aligned} P_{C \omega}=p_{C \omega} \frac{H}{A \overline{T_{s}} \lambda} & \\
& =\frac{H_{h}}{4} \int_{\frac{1}{4 F o}}^{\infty} \frac{T_{s}\left(t-\frac{H^{2}}{4 a \varphi}\right)}{\overline{T_{s}}}\left\{\frac{-4 Z}{\sqrt{\pi \varphi}} \exp \left[-Z^{2} \varphi\right]\right. \\
& -\left(\frac{H_{h}}{\varphi}\right)^{2} \exp \left(Z H_{h}+\frac{H_{h}{ }^{2}}{4 \varphi}\right)\left[\operatorname{erfc}\left(Z \sqrt{\varphi}+\frac{H_{h}}{2 \sqrt{\varphi}}\right)\right] \\
& \left.+2 \sqrt{\frac{\varphi}{\pi}} \exp \left[Z H_{h}+\frac{H_{h}{ }^{2}}{4 \varphi}-\left(Z \sqrt{\varphi}+\frac{H_{h}}{2 \sqrt{\varphi}}\right)^{2}\right]\right\} d \varphi\end{aligned}$ & $\mathrm{C} 13$ \\
\hline$(21)^{*}$ & $\theta_{D}=\Omega_{D}+\mathrm{B}_{M F L S}$ & $\mathrm{C} 14$ \\
\hline (22) & $\Omega_{D}=\frac{Z}{4 \sqrt{\pi}} \int_{\frac{1}{4 F o}}^{\infty} \frac{T_{s}\left(t-\frac{H^{2}}{4 a \varphi}\right)}{\overline{T_{s}} \sqrt{\varphi}} \exp \left[-Z^{2} \varphi\right] \Delta(\boldsymbol{X}, \varphi) d \varphi$ & C15 \\
\hline (23) & $\begin{aligned} F_{D \beta}=f_{D \beta} \frac{H}{T_{S} \lambda} & =\frac{Q_{T}}{2 \mathrm{R} \pi^{3 / 2}} \exp \left(\frac{X P e}{2}\right) \int_{\frac{\mathrm{R}^{2}}{4 F o}}^{\infty} \frac{1}{\sqrt{\varphi}} \exp \left(-\varphi-\left(\frac{R P e}{2}\right)^{2} \frac{1}{\varphi}\right)[1 \\
& \left.-\exp \left(-\frac{\varphi}{\mathrm{R}^{2}}\right)\right] d \varphi\end{aligned}$ & $\mathrm{C} 16$ \\
\hline (24) & $\begin{aligned} & F_{D \omega}=f_{D \omega} \frac{H}{\overline{T_{s}} \lambda} \\
&=\frac{1}{4 \sqrt{\pi}} \int_{\frac{1}{4 F o}}^{\infty} \frac{T_{s}\left(t-\frac{H^{2}}{4 a \varphi}\right)}{\overline{T_{s}} \sqrt{\varphi}} \exp \left[-Z^{2} \varphi\right][1-2 \\
&\left.\cdot Z^{2} \varphi\right] \exp \left[-Z^{2} \varphi\right] \Delta(\boldsymbol{X}, \varphi) d \varphi\end{aligned}$ & $\mathrm{C} 17$ \\
\hline (25) & $F_{D T}=F_{D \omega}+F_{D \beta}$ & $\mathrm{C} 18$ \\
\hline (26) & $P_{D \beta}=\frac{p_{D \beta}}{q H}=\left[\sqrt{\frac{4 F o}{\pi}}+\operatorname{erf}\left(-\frac{1}{\sqrt{4 F o}}\right)-\sqrt{\frac{4 F o}{\pi}} \exp \left(-\frac{1}{4 F o}\right)+1\right]$ & C19 \\
\hline (27) & $p_{D \omega}=P_{D \omega} \frac{H}{A \overline{T_{s}} \lambda}=\frac{1}{\sqrt{\pi}} \int_{\frac{1}{4 F o}}^{\infty} \frac{T_{s}\left(t-\frac{H^{2}}{4 a \varphi}\right)}{\overline{T_{s}} \sqrt{\varphi}} \exp \left[-Z^{2} \varphi\right]\left[1-2 \cdot Z^{2} \varphi\right] d \varphi$ & $\mathrm{C} 20$ \\
\hline
\end{tabular}




$$
P_{D T}=P_{D \beta}+P_{D \omega}
$$




\section{References}

1. Menberg, K., et al., Long-Term Evolution of Anthropogenic Heat Fluxes into a Subsurface Urban Heat Island. Environmental science \& technology, 2013. 47(17): p. 9747-9755.

2. Ferguson, G. and A.D. Woodbury, Subsurface heat flow in an urban environment. Journal of Geophysical Research: Solid Earth (1978-2012), 2004. 109(B2).

3. Zhu, K., et al., The geothermal potential of urban heat islands. Environmental Research Letters, 2010. 5(4): p. 044002.

4. Herbert, A., S. Arthur, and G. Chillingworth, Thermal modelling of large scale exploitation of ground source energy in urban aquifers as a resource management tool. Applied Energy, 2013. 109: p. 94-103.

5. Mirzaei, P.A. and F. Haghighat, Approaches to study urban heat island-abilities and limitations. Building and Environment, 2010. 45(10): p. 2192-2201.

6. $\quad$ Kollet, S.J., et al., The influence of rain sensible heat and subsurface energy transport on the energy balance at the land surface. Vadose Zone Journal, 2009. 8(4): p. 846-857.

7. Liu, C., et al., A numerical and field Investigation of underground temperatures under urban heat island. Building and Environment, 2011. 46(5): p. 1205-1210.

8. Errera, M., S. Lorente, and A. Bejan, Assemblies of heat pumps served by a single underground heat exchanger. International Journal of Heat and Mass Transfer, 2014. 75: p. 327-336.

9. Zhu, K., et al., Groundwater temperature evolution in the subsurface urban heat island of Cologne, Germany. Hydrological Processes, 2014.

10. García-Gil, A., et al., The thermal consequences of river-level variations in an urban groundwater body highly affected by groundwater heat pumps. Science of The Total Environment, 2014. 485486(0): p. 575-587.

11. Taylor, C.A. and H.G. Stefan, Shallow groundwater temperature response to climate change and urbanization. Journal of Hydrology, 2009. 375(3): p. 601-612.

12. Benz, S.A., et al., Spatial resolution of anthropogenic heat fluxes into urban aquifers. Science of The Total Environment, 2015. 524-525(0): p. 427-439.

13. Ferguson, G., Screening for heat transport by groundwater in closed geothermal systems. Groundwater, 2015. 53(3): p. 503-506.

14. Yang, H., P. Cui, and Z. Fang, Vertical-borehole ground-coupled heat pumps: a review of models and systems. Applied Energy, 2010. 87(1): p. 16-27.

15. Stauffer, F., et al., Thermal use of shallow groundwater. 2013: CRC Press.

16. Kavanaugh, S.P. and K.D. Rafferty, Ground-source heat pumps: design of geothermal systems for commercial and institutional buildings. 1997: American Society of Heating, Refrigerating and AirConditioning Engineers.

17. Lamarche, L. and B. Beauchamp, New solutions for the short-time analysis of geothermal vertical boreholes. International Journal of Heat and Mass Transfer, 2007. 50(7-8): p. 1408-1419.

18. Tye-Gingras, M. and L. Gosselin, Generic ground response functions for ground exchangers in the presence of groundwater flow. Renewable Energy, 2014. 72: p. 354-366.

19. de Paly, M., et al., Optimization of energy extraction for closed shallow geothermal systems using linear programming. Geothermics, 2012. 43: p. 57-65.

20. Erol, S., M.A. Hashemi, and B. François, Analytical solution of discontinuous heat extraction for sustainability and recovery aspects of borehole heat exchangers. International Journal of Thermal Sciences, 2015. 88: p. 47-58.

21. Molina-Giraldo, N., P. Bayer, and P. Blum, Evaluating the influence of thermal dispersion on temperature plumes from geothermal systems using analytical solutions. International Journal of Thermal Sciences, 2011. 50(7): p. 1223-1231.

22. Bayer, P., M. de Paly, and M. Beck, Strategic optimization of borehole heat exchanger field for seasonal geothermal heating and cooling. Applied Energy, 2014. 136: p. 445-453.

23. Ingersoll, L., O. Zobel, and A. Ingersoll, Heat Conduction with Engineering, Geological and Other Applications. 1954, New York Mcgraw-Hill.

24. Carslaw, H. and J. Jaeger, Conduction of Heat in Solids. second ed. 1959, New York: Oxford University Press. 
25. Li, M. and A.C.K. Lai, Review of analytical models for heat transfer by vertical ground heat exchangers (GHEs): A perspective of time and space scales. Applied Energy, 2015. 151(0): p. 178191.

26. Zeng, H., N. Diao, and Z. Fang, A finite line-source model for boreholes in geothermal heat exchangers. Heat Transfer-Asian Research, 2002. 31(7): p. 558-567.

27. Molina-Giraldo, N., et al., A moving finite line source model to simulate borehole heat exchangers with groundwater advection. International Journal of Thermal Sciences, 2011. 50(12): p. 2506-2513.

28. Bandos, T., et al., Finite line-source model for borehole heat exchangers: effect of vertical temperature variations. Geothermics, 2009. 38(2): p. 263-270.

29. Rivera, J.A., P. Blum, and P. Bayer, Analytical simulation of groundwater flow and land surface effects on thermal plumes of borehole heat exchangers. Applied Energy, 2015. 146(0): p. 421-433.

30. Signorelli, S. and T. Kohl, Regional ground surface temperature mapping from meteorological data. Global and Planetary Change, 2004. 40(3): p. 267-284.

31. Cheon, J.-Y., et al., Soil temperatures in four metropolitan cities of Korea from 1960 to 2010: implications for climate change and urban heat. Environmental earth sciences, 2014. 71(12): p. 5215-5230.

32. Dědeček, P., et al., Six years of ground-air temperature tracking at Malence (Slovenia): thermal diffusivity from subsurface temperature data. Journal of Geophysics and Engineering, 2013. 10(2): p. 025012.

33. Duan, X. and G.F. Naterer, Ground Heat Transfer From a Varying Line Source With Seasonal Temperature Fluctuations. Journal of Heat Transfer, 2008. 130(11): p. 111302-111302.

34. Kurylyk, B.L. and K.T. MacQuarrie, A new analytical solution for assessing climate change impacts on subsurface temperature. Hydrological Processes, 2013.

35. Zhan, W., et al., Satellite-derived Subsurface Urban Heat Island. Environmental science \& technology, 2014.

36. Smerdon, J.E., et al., Air-ground temperature coupling and subsurface propagation of annual temperature signals. Journal of Geophysical Research: Atmospheres (1984-2012), 2004. 109(D21).

37. Stieglitz, M. and J.E. Smerdon, Characterizing land-atmosphere coupling and the implications for subsurface thermodynamics. Journal of climate, 2007. 20(1): p. 21-37.

38. Brookfield, A., et al., Thermal transport modelling in a fully integrated surface/subsurface framework. Hydrological processes, 2009. 23(15): p. 2150-2164.

39. Krarti, M., et al., Analytical model to predict annual soil surface temperature variation. Journal of solar energy engineering, 1995. 117(2): p. 91-99.

40. Cleall, P.J., J.J. Muñoz-Criollo, and S.W. Rees, Analytical Solutions for Ground Temperature Profiles and Stored Energy Using Meteorological Data. Transport in Porous Media, 2015. 106(1): p. 181-199.

41. Güven, O., J. Melville, and F. Molz, An analysis of the effect of surface heat exchange on the thermal behavior of an idealized aquifer thermal energy storage system. Water Resources Research, 1983. 19(3): p. 860-864.

42. Claesson, J. and C.-E. Hagentoft, Heat loss to the ground from a building-I. General theory. Building and Environment, 1991. 26(2): p. 195-208.

43. Hagentoft, C.-E., Heat loss to the ground from a building. Slab on the ground and cellar, Lund Institute of Technology, Report TVBH-1004, Lund, 1988.

44. Mihalakakou, G., et al., On the application of the energy balance equation to predict ground temperature profiles. Solar Energy, 1997. 60(3): p. 181-190.

45. Tsilingiridis, G. and K. Papakostas, Investigating the relationship between air and ground temperature variations in shallow depths in northern Greece. Energy, 2014. 73: p. 1007-1016.

46. Ouzzane, M., et al., Analysis of the convective heat exchange effect on the undisturbed ground temperature. Solar Energy, 2014. 108: p. 340-347.

47. Fujii, H., et al., Numerical simulation and sensitivity study of double-layer Slinky-coil horizontal ground heat exchangers. Geothermics, 2013. 47: p. 61-68.

48. Rivera, J.A., P. Blum, and P. Bayer, A finite line source model with Cauchy-type top boundary conditions for simulating near surface effects on borehole heat exchangers. Energy Journal, in revision.

49. Özış1k, M.N., Boundary value problems of heat conduction. 1989: Courier Corporation.

50. Diao, N., Q. Li, and Z. Fang, Heat transfer in ground heat exchangers with groundwater advection. International Journal of Thermal Sciences, 2004. 43(12): p. 1203-1211. 
51. Rivera, J.A., P. Blum, and P. Bayer, Ground energy balance for borehole heat exchangers: Vertical fluxes, groundwater and storage. Renewable Energy, 2015. 83(0): p. 1341-1351.

52. Janssen, H., J. Carmeliet, and H. Hens, The influence of soil moisture in the unsaturated zone on the heat loss from buildings via the ground. Journal of Building Physics, 2002. 25(4): p. 275-298.

53. Diersch, H.-J., FEFLOW: Finite element modeling of flow, mass and heat transport in porous and fractured media. 2013: Springer Science \& Business Media.

54. Deng, J.S., et al., Spatio-temporal dynamics and evolution of land use change and landscape pattern in response to rapid urbanization. Landscape and Urban Planning, 2009. 92(3): p. 187-198.

55. Li, C. and N.X. Thinh, Investigation and comparison of land-cover change patterns in Xuzhou city, China, and Dortmund city region, Germany, using multitemporal Landsat images. Journal of Applied Remote Sensing, 2013. 7(1): p. 073458-073458.

56. Bayer, P., et al., Extracting past atmospheric warming and urban heating effects from borehole temperature profiles. Geothermics, under review.

57. Dědeček, P., J. Šafanda, and D. Rajver, Detection and quantification of local anthropogenic and regional climatic transient signals in temperature logs from Czechia and Slovenia. Climatic change, 2012. 113(3-4): p. 787-801.

58. Rybach, L., Classification of geothermal resources by potential. Geothermal Energy Science, 2015. 3: p. 13-17. 